\title{
Molecular phylogeny of Cytospora species associated with canker diseases of fruit and nut crops in California, with the descriptions of ten new species and one new combination
}

\author{
Daniel P. Lawrence ${ }^{1}$, Leslie A. Holland ${ }^{1}$, Mohamed T. Nouri², Renaud Travadon ${ }^{1}$, Ara Abramians ${ }^{1}$, Themis J. Michailides², and \\ Florent P. Trouillas ${ }^{2}$
}

${ }^{1}$ Department of Plant Pathology, University of California, Davis, One Shields Avenue, Davis, CA 95616, USA

${ }^{2}$ Department of Plant Pathology, University of California, Davis and Kearney Agricultural Research and Extension Centre, Parlier, CA 93648, USA; corresponding author e-mail: flotrouillas@ucdavis.edu

\begin{abstract}
Cytospora species are destructive canker and dieback pathogens of woody hosts in natural and agroecosystems around the world. In this genus, molecular identification has been limited due to the paucity of multi-locus sequence typing studies and the lack of sequence data from type specimens in public repositories, stalling robust phylogenetic reconstructions. In most cases a morphological species concept could not be applied due to the plasticity of characters and significant overlap of morphological features such as spore dimensions and fruiting body characters. In this study, we employed a molecular phylogenetic framework with the inclusion of four nuclear loci (ITS, translation elongation factor 1-alpha, actin, and beta-tubulin) to unveil the biodiversity and taxonomy of this understudied important genus of plant pathogens. Phylogenetic inferences based on 150 Californian isolates revealed 15 Cytospora species associated with branch and twig cankers and dieback of almond, apricot, cherry, cottonwood, olive, peach, pistachio, plum, pomegranate, and walnut trees in California. Of the 15 species recovered in this study, 10 are newly described and typified, in addition to one new combination. The pathogenic status of the newly described Cytospora species requires further investigation as most species were associated with severe dieback and decline of diverse and economically important fruit and nut crops in California.
\end{abstract}

Key words:

Cytosporaceae

Cytospora canker

Diaporthales

multigene phylogeny

new taxa

taxonomy

Article info: Submitted: 30 March 2018; Accepted: 12 September 2018; Published: 26 September 2018.

\section{INTRODUCTION}

The generic name Cytospora (Sordariomycetes, Diaporthales, Cytosporaceae) was introduced in 1818 and includes seemingly innocuous endophytes isolated from the bark, xylem, and leaves of asymptomatic woody plants (Spielman 1983, Bills 1996), saprobes that colonize and degrade the wood of dead or dying trees (Christensen 1940), and destructive canker pathogens (known as Cytospora-, Leucostoma-, Valsa-, or perennial canker) that cause dieback of more than 85 woody plant species (Sinclair et al. 1987, Adams et al. 2005, 2006). The chronic wood infections caused by Cytospora species can be devastating to stone fruit, pome fruit, and nut crops such as Prunus persica, $P$. armeniaca, P. avium, Malus spp., and Juglans spp. (Biggs \& Grove 2005, Wang et al. 2011, Fan et al. 2015a). Cytospora species mainly impact branches, but they can cause more destructive infections in the trunks and larger scaffolds, severely limiting the longevity and productivity of orchards (Biggs 1989, Chang et al. 1991).

To date, approximately 612 Cytospora species have been described according to Index Fungorum. Kirk et al. (2008) listed approximately 110 accepted Cytospora species, while all other species names were considered synonyms of previously described taxa or treated as non-Cytospora species before the one fungus = one name rule came into force in July 2011 (Hawksworth 2011). Therefore, all taxa including the former sexual and asexual morphs that no longer have nomenclatural priority should be considered in order to resolve nomenclatural issues in this group of challenging fungi. The asexual morph is commonly encountered in nature. The pycnidia arise in a stroma embedded in host tissues (Grove 1923), and possess either a single locule or a complex of invaginated walls producing labyrinthine locules with filamentous conidiophores which may be reduced to conidiogenous cells that bear hyaline, allantoid conidia (Adams et al. 2006). Pycnidia exude conidia in a yellow, orange to red polysaccharide matrix, a cirrus, via an ostiole (Adams et al. 2005, 2006). Conidia oozing from pycnidia embedded in dead or dying host cortical tissues during humid or wet conditions are considered the infectious propagules potentially initiating new infections; the role of ascospores has not been determined. Conidia are dispersed to new plant tissues by rain-splash, where they germinate and infect the host plant via cracks and wounds to the bark created by pruning wounds, leaf scars, insect injuries,

๑) 2018 International Mycological Association

You are free to share - to copy, distribute and transmit the work, under the following conditions:

Attribution: $\quad$ You must attribute the work in the manner specified by the author or licensor (but not in any way that suggests that they endorse you or your use of the work).

Non-commercial: $\quad$ You may not use this work for commercial purposes.

No derivative works: You may not alter, transform, or build upon this work.

For any reuse or distribution, you must make clear to others the license terms of this work, which can be found at http://creativecommons.org/licenses/by-nc-nd/3.0/legalcode. Any of the above conditions can be waived if you get permission from the copyright holder. Nothing in this license impairs or restricts the author's moral rights. 
winter-injured buds, twigs and bark, and breakage of shadeweakened twigs and branches (Tekauz \& Patrick 1974, Biggs 1989). Bertrand \& English (1976b) showed that Cytospora conidia were routinely trapped up to $76.8 \mathrm{~m}$ from the primary inoculum source after wind-blown rain in California, thus providing compelling evidence for Cytospora spore dispersal across large areas within orchards during times of inclement weather.

Species diagnosis in Cytospora has traditionally relied on morphological characters of pycnidia/perithecia (Grove 1923), including locule shape/organization and spore dimensions (Spielman 1985), as well as the arrangement of stromatic tissues (Adams et al. 2002). This morphological species approach is confounded by many examples of morphological character overlap among species and by the morphological plasticity of pycnidial locules which are affected by the host bark and cambium characteristics (Adams et al. 2002, Wang et al. 2011). Species diagnosis based on host association has also proven unreliable as several species of Cytospora have been recovered from multiple distantly related hosts, while a single host species can harbour more than one species of Cytospora (Adams et al. 2005, 2006, Wang et al. 2011, Fan et al. 2015a, b).

Défago (1935) questioned the utility of morphological characters in delimiting Cytospora species. Spielman (1985) reported that the asexual morph of Cytospora leucosperma was indistinguishable from that of many other species of Cytospora. Traditionally, sexual morphs of Cytospora were classified within several genera including Leucostoma, Valsa, Valsella, and Valseutypella. Tulasne \& Tulasne (1863) postulated that Cytospora and Valsa were the asexual and sexual morphs of the same fungus. All these studies have highlighted the difficulty to properly disentangle taxa that share similar morphologies. Species identification based on molecular data could overcome these difficulties, which has been illustrated using ITS rDNA phylogenies (Adams et al. 2002, 2005, 2006). Recently, the use of the generic name Cytospora has been recommended for protection and use over Leucocytospora, Leucostoma, Valsa, Valsella, and Valseutypella (Rossman et al. 2015).

According to Norphanphoun et al. (2017) there are currently only 23 ex-type Cytospora species sequences deposited in GenBank. The majority of these sequences correspond to a single nuclear ribosomal gene region covering the ITS or the partial nuclear large ribosomal RNA subunit (nrLSU). Molecular data from type specimens are thus limited in public repositories and hamper abilities to properly circumscribe or identify taxa to the species-level in Cytospora. Recently, the utility of additional protein-coding loci, such as beta-tubulin, actin, and translation elongation factor 1-alpha, has been demonstrated for Cytospora sequence-based identification: more Cytospora species were recognized when using analyses including multiple protein-coding loci, relative to analyses relying on ITS only or combined ITS and nrLSU (Lawrence et al. 2017a).
Although Cytospora species are known pathogens of stone fruits and nut crops worldwide, the taxonomy and host distribution of Cytospora species occurring in California orchards are still elusive, with only C. leucostoma and/or C. cincta known to affect French prune (Bertrand \& English 1976a), peach and nectarine (French 1989), and sweet cherry (Trouillas et al. 2012). California is the largest and most productive perennial agricultural area in North America, producing diverse fruit and nut crops which constitute potential hosts for Cytospora species. The objectives of this study were to examine the phylogenetic diversity of Cytospora species isolated from orchards exhibiting dieback and canker diseases in California. Our hypotheses were that new Cytospora species would be identified from a region and crops that have been under-examined, especially given the recent advances in molecular identification of fungi (Hibbett et al. 2016). We hypothesized also that distinct species of Cytospora would infect distinct crop species, as expected if host specificity would favour pathogen speciation (Giraud et al. 2006). Morphological characters in conjunction with multilocus phylogenetic analyses will afford us the first glimpse into the biodiversity of this important genus of canker pathogens.

\section{MATERIALS AND METHODS}

\section{Fruit and nut crop sampling and fungal isolation}

Between 2010 and 2017, putative Cytospora species were isolated periodically from declining fruit and nut trees throughout the Central Valley region of California as part of the diagnosis activity of the co-operative extension laboratories at the Kearney Agricultural Research and Extension Centre, in the centre of major agricultural industries. Sampled trees expressed general symptoms and signs of canker diseases including branch dieback, leaf wilting, dead and split bark, sunken lesions on branches, internal wood discoloration, gumming on trunks and scaffold limbs, cracked bark revealing blackened tissues, and presence of pinhead-sized dark pycnidia erupting through the bark or exposed upon peeling the outer layer of the bark (Figs 1-3). Mass-hyphal isolates were recovered using 10-12 wood pieces $(4 \times 4$ $\times 2 \mathrm{~mm}$ ) per sample, cut from the margins of necrotic and apparently healthy wood, surface disinfested in $0.6 \%$ sodium hypochlorite for $60 \mathrm{~s}$, rinsed in two serial baths of sterile deionized water for $30 \mathrm{~s}$, and plated on potato dextrose agar (PDA, Difco, Detroit, MI) dishes amended with tetracycline (1 $\left.\mathrm{mg} \mathrm{L}^{-1}\right)$. A number of isolates were also collected directly from conidial masses exuding from freshly exposed pycnidia on declining branches. Masses of conidia were collected using a sterilized needle, placed into $1.5 \mathrm{~mL}$ tubes containing sterile water, and spread onto the surface of PDA Petri dishes. Petri dishes were incubated at $25{ }^{\circ} \mathrm{C}$ in the dark for up to $28 \mathrm{~d}$. Isolates with morphological characters of Cytospora, namely

Fig. 1. Signs and symptoms of Cytospora canker/dieback in various fruit and nut crops in California. A. Twig dieback in sweet cherry. B. Twig and scaffold branch dieback in French prune. C. Pimpled-bark indicating underlying asexual fruiting bodies in a sweet cherry branch affected with Cytospora canker. D. Below bark, asexual fruiting bodies associated with Cytospora canker of French prune. E-F. Cankers and wood discoloration associated with Cytospora canker of sweet cherry. 

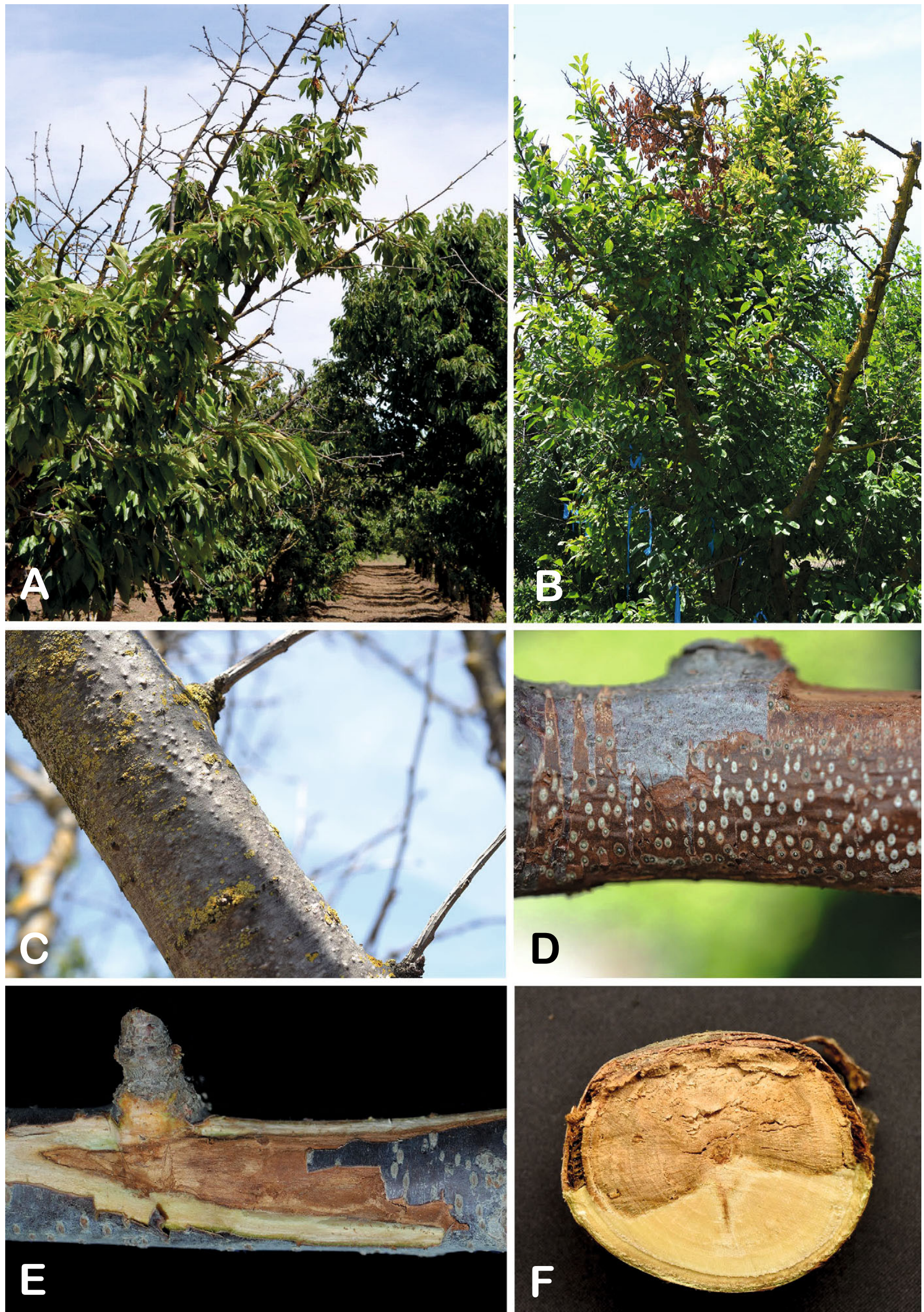

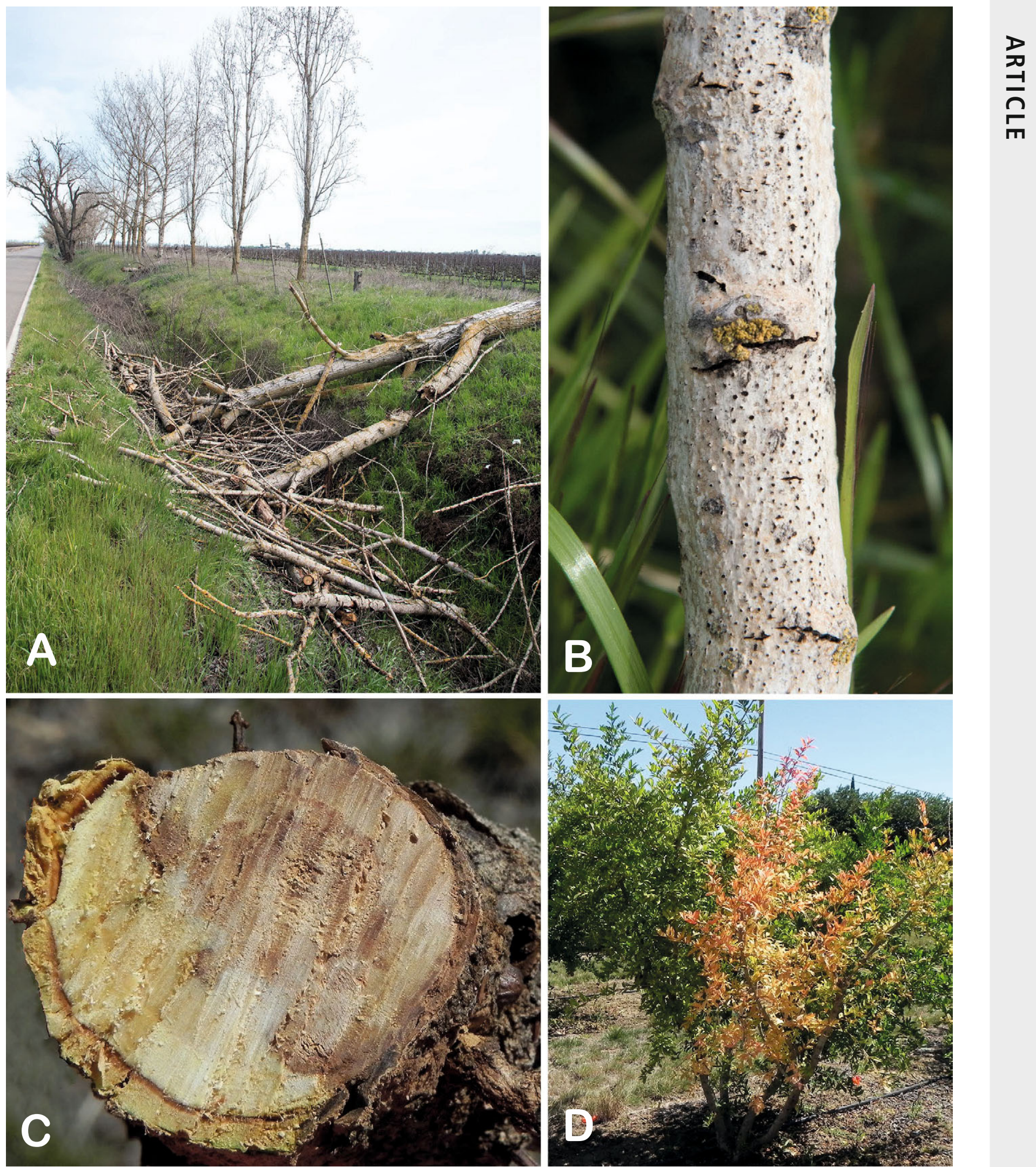

Fig. 3. Signs and symptoms of Cytospora canker/dieback in cottonwood and pomegranate hosts in California. A-B. Dead cottonwood tree parts on a roadside surrounding orchards and associated Cytospora asexual fruiting bodies erupting through the bark. C-D. Cytospora canker, wood discoloration and associated branch dieback in pomegranate.

Fig. 2. Signs and symptoms of Cytospora canker/dieback in various fruit and nut crops in California. A-B. Gumming and underlying elongated canker associated with Cytospora canker in almond. C. Cytospora associated cankers in olive twigs. D. Cankers and wood discoloration associated with Cytospora in pistachio. E. Conidial masses exuding from Cytospora asexual fruiting bodies in walnut. 
colonies with uneven to highly uneven growth margins and thus lobate to highly lobate colony morphology, were hyphal-tip purified to fresh PDA dishes. In total, 150 isolates from symptomatic orchards and adjacent ornamental trees throughout the Central Valley of California were recovered in pure culture and used for phylogenetic and morphological analyses (Table 1). Representative cultures used in this study are permanently preserved in the collections of the Department of Plant Pathology at the Kearney Agricultural Research and Extension Centre of the University of California, Parlier, CA. The holotypes of the newly described species are preserved as dried cultures in BPI, with ex-type cultures deposited in CBS.

\section{DNA extraction, sequencing, and phylogenetic analyses}

Total genomic DNA was isolated from mycelium scraped with a sterile scalpel from the surface of 14-day-old cultures using the DNeasy Plant kit (Qiagen, Valencia, CA), following the manufacturer's instructions. All PCR reactions utilized AccuPower ${ }^{\mathrm{TM}}$ PCR Premix (Bioneer, Alameda, CA), following the manufacturer's instructions. Amplification of rDNA, including the intervening ITS regions and 5.8S rDNA (ITS15.8S-ITS2), using the primer set ITS5 and ITS4 followed the protocol of White et al. (1990). Amplification of translation elongation factor 1- $\alpha$ (TEF1) fragments utilized the primer set EF1-688F and EF1-1251R (Alves et al. 2008), beta-tubulin (TUB2) utilized primers Bt1a and Bt1b (Glass \& Donaldson 1995), and actin (ACT1) utilized primers ACT-512F and ACT-783R (Carbone \& Kohn 1999), with a slightly modified PCR program for TUB2 and ACT1 [initial denaturation (94 ${ }^{\circ} \mathrm{C}, 5 \mathrm{~min}$ ) followed by 35 cycles of denaturation $\left(94{ }^{\circ} \mathrm{C}, 30\right.$ $\mathrm{s})$, annealing $\left(58{ }^{\circ} \mathrm{C}\right.$ for TUB2 and $63{ }^{\circ} \mathrm{C}$ for ACT1, $\left.30 \mathrm{~s}\right)$, extension $\left(72^{\circ} \mathrm{C}, 60 \mathrm{~s}\right)$, and a final extension $\left.\left(72^{\circ} \mathrm{C}, 10 \mathrm{~min}\right)\right]$. PCR amplification of the TUB2 locus for some Californian Cytospora isolates (described below) was attempted at different annealing temperatures $\left(50-60{ }^{\circ} \mathrm{C}\right)$. PCR products were visualized on a $1.5 \%$ agarose gel $(120 \mathrm{~V}$ for $25 \mathrm{~min}$ ) stained with GelRed $\AA$ (Biotium, Fremont, CA), following the manufacturer's instructions, to confirm presence and size of amplicons, purified via Exonuclease I and recombinant Shrimp Alkaline Phosphatase (Affymetrix, Santa Clara, CA), and sequenced bidirectionally via BigDye $\AA$ Terminator v. 3.1 Cycle Sequencing Kit (Thermo Fischer Scientific, Waltham, $\mathrm{MA})$ on an $\mathrm{ABI} 3730$ Capillary Electrophoresis Genetic Analyzer (College of Biological Sciences Sequencing Facility, University of California, Davis).

Forward and reverse nucleotide sequences were assembled, proofread, and edited in Sequencher v. 5 (Gene Codes Corporation, Ann Arbor, MI) and deposited in GenBank (Table 1). Homologous sequences with high similarity from ex-type and non-type Cytospora isolates were included for phylogenetic reference utilizing the BLASTn function in NCBI and extensive literature review (Table 2). Multiple sequence alignments were performed in MEGA v. 6 (Tamura et al. 2013) and manually adjusted where necessary in Mesquite v. 3.10 (Maddison \& Maddison 2016). Alignments were submitted to TreeBASE under accession number S22195. Phylogenetic analyses were performed for each individual locus and for a four-gene concatenated dataset. Each dataset was analyzed using two different optimality search criteria, maximum parsimony (MP) and maximum likelihood (ML), in MEGA v. 6 (Tamura et al. 2013). For MP analyses, heuristic searches with 1000 random sequence additions were implemented with the Tree-Bisection-Reconnection algorithm, gaps were treated as missing data. Bootstrap analyses with 1000 pseudoreplicates were used to estimate branch support. For ML analyses, MEGA was used to infer a model of nucleotide substitution for each dataset, using the Akaike Information Criterion (AIC). All ML analyses utilized the Nearest-Neighbor-Interchange heuristic method and branch support was determined by 1000 bootstrap pseudoreplicates. Sequences of Diaporthe ampelina isolate Wolf912 and $D$. benedicti isolate SBen914 (Diaporthales, Diaporthaceae) (Lawrence et al. 2015) served as the outgroup taxa in all analyses.

\section{Morphology}

Mycelial plugs (5 $\mathrm{mm}$ diam) were taken from the margin of selected, actively growing cultures based on preliminary phylogenetic results and transferred to triplicate $90 \mathrm{~mm}$ diam Petri dishes containing $2 \%$ PDA and incubated in the dark at $25^{\circ} \mathrm{C}$ for $14 \mathrm{~d}$. Radial growth was measured after $7 \mathrm{~d}$ by taking two measurements perpendicular to each other. Assessments of colony colour (Rayner 1970) and morphology were made after $14 \mathrm{~d}$. Pycnidia were induced on corticated cherry wood embedded in PDA medium. Cherry cuttings (approx. $1 \mathrm{~cm}$ diam) were collected and cut into $5 \mathrm{~cm}$ sections. Sections were placed in glass Petri dishes and autoclaved twice, $24 \mathrm{~h}$ apart, at $122{ }^{\circ} \mathrm{C}$ for $25 \mathrm{~min}$. Autoclaved wood sections were placed in $90 \mathrm{~mm}$ diam plastic Petri dishes, two sections per dish, and PDA was poured to embed them. A mycelial plug from an actively growing culture was placed between the two wood sections in each dish, one isolate per dish. Petri dishes were incubated at room temperature under natural photoperiod in August 2017, and pycnidial formation was monitored weekly for four weeks. Morphological characterization of the pycnidia $(n=20)$ included the diameter, presence/absence of a conceptacle, and colour using a binocular Leica MZ95 dissecting microscope (Leica microsystems CMS, Wetzlar, Germany). Pycnidial locular arrangements were assessed by transversely sectioning pycnidia by hand with a razor blade and observing as above. Conidial dimensions $(n=30)$ and conidiogenous cells $(n=20)$ were measured at $\times 1000$ from approximately 28 -day-old cultures by producing a pycnidial squash mount that was crushed in a sterile $50 \%$ glycerol solution (no stain was applied, thus the natural pigments of each species was preserved) and observed with a Leica DM500B microscope (Leica microsystems CMS, Wetzlar, Germany). Morphological measurements are represented by the mean as a range depicting the standard deviation in the centre with minima and maxima in parentheses, respectively, in the species descriptions and taxonomy section below.

\section{RESULTS}

\section{Disease symptoms, hosts, and distribution}

In total, 92 samples were obtained from symptomatic trees in 70 orchards of various fruit and nut crops including almond (Prunus dulcis), apricot (Prunus armeniaca), cherry (Prunus 


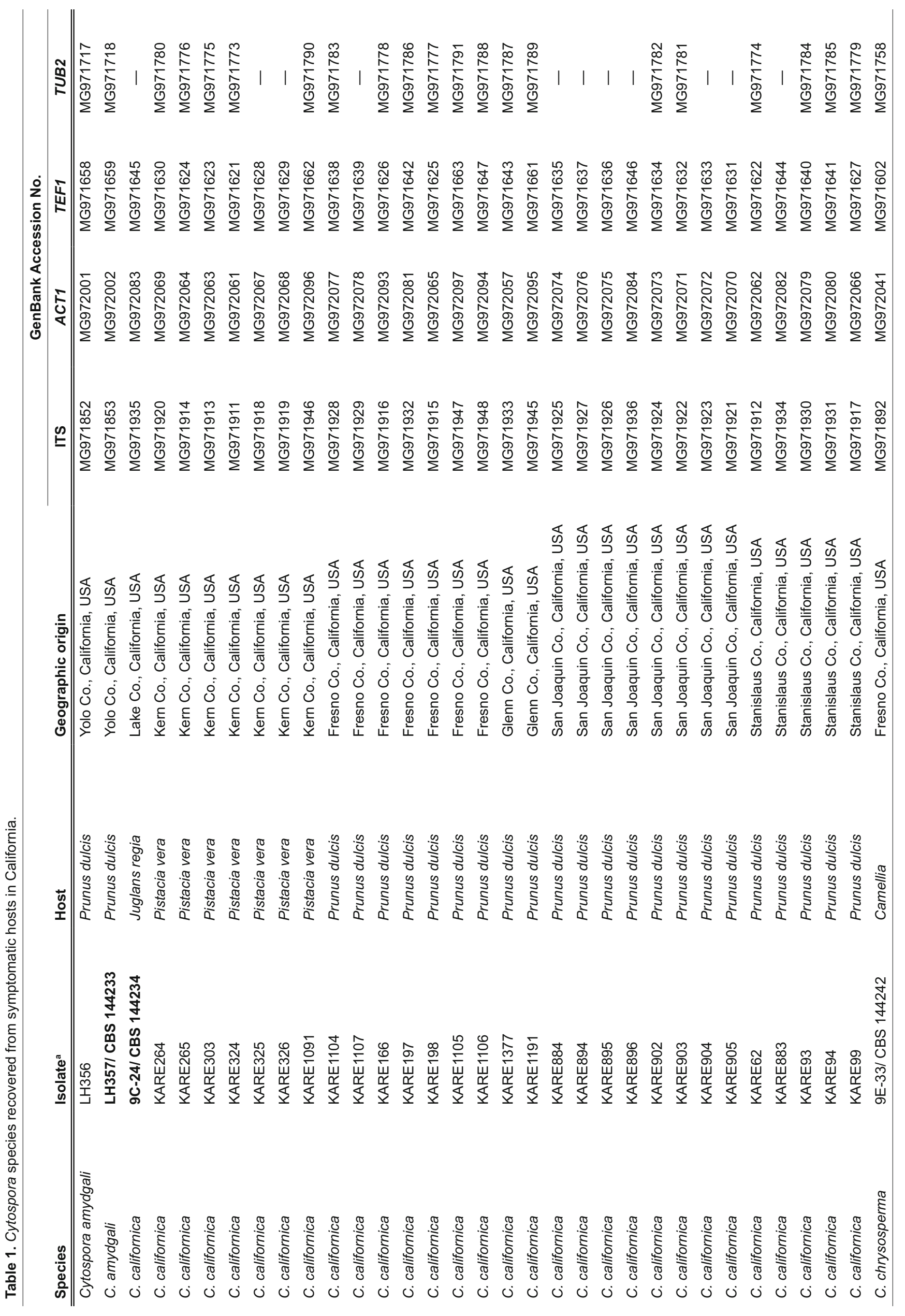




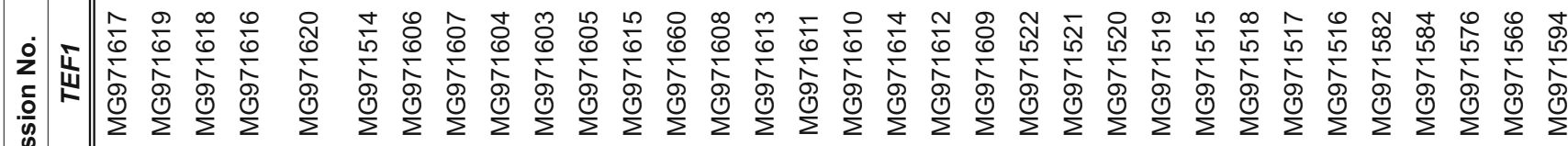

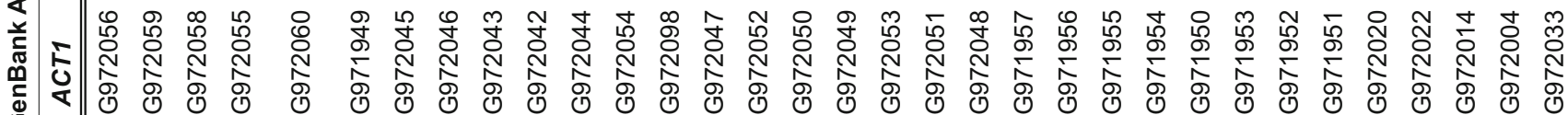

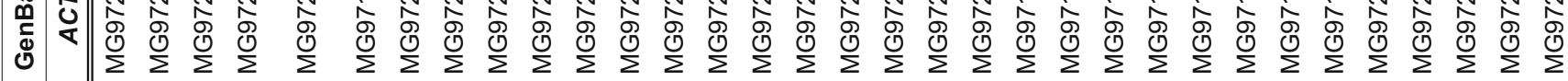

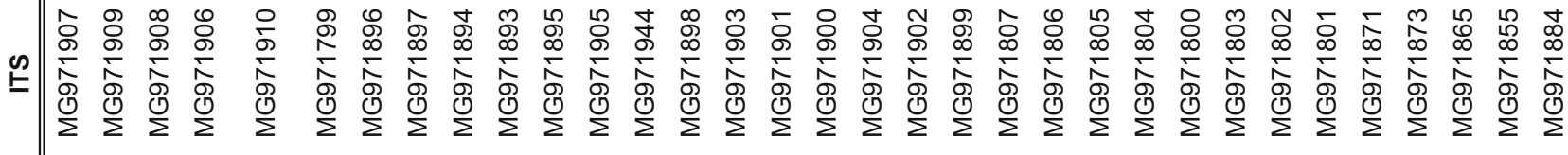

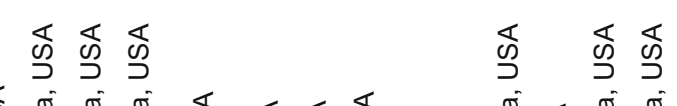

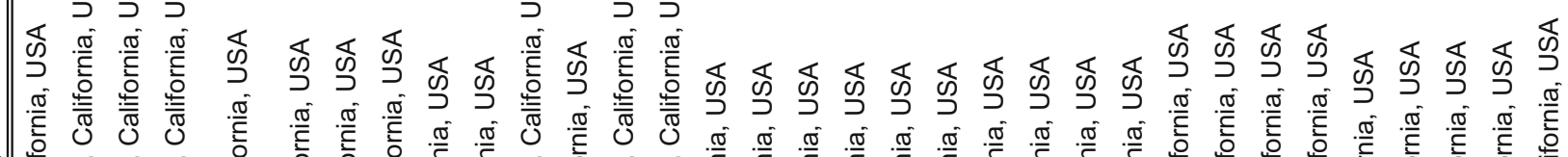

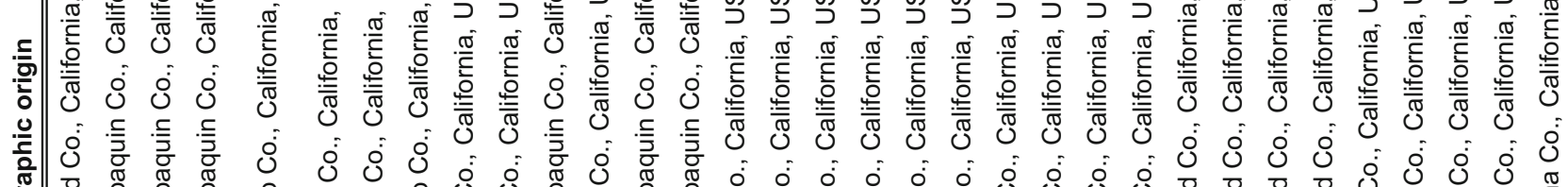

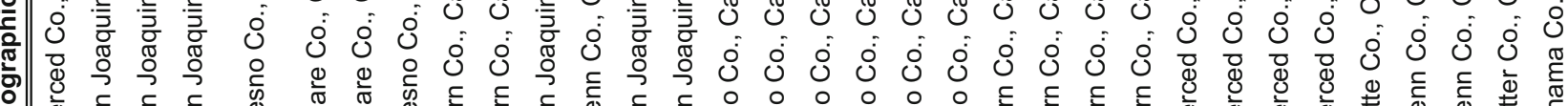

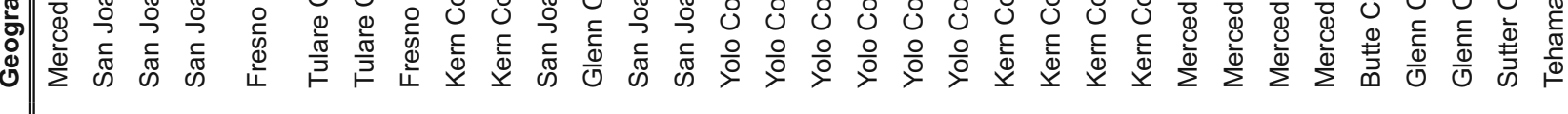

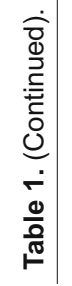

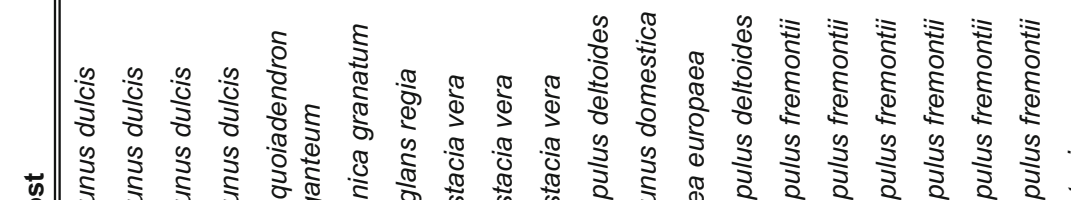

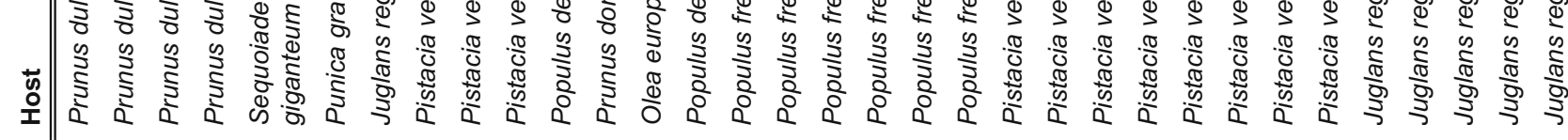

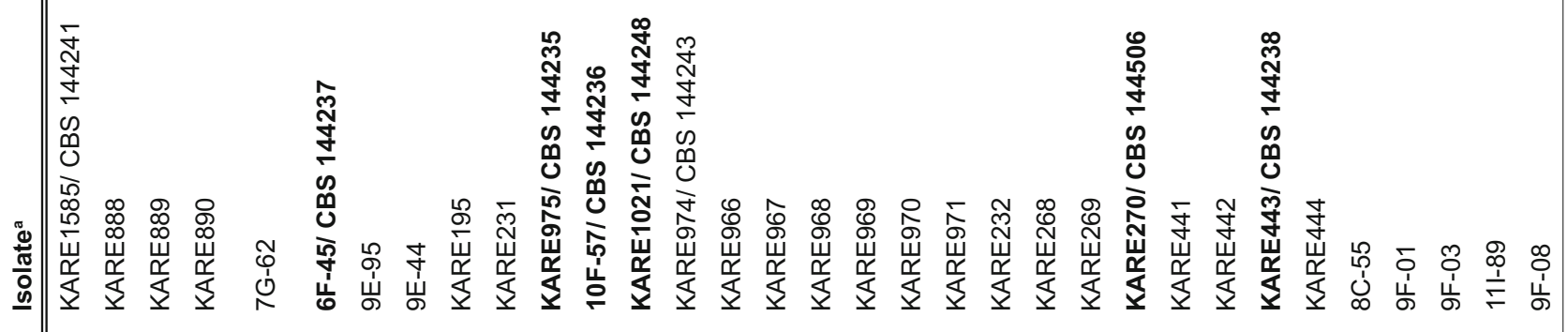




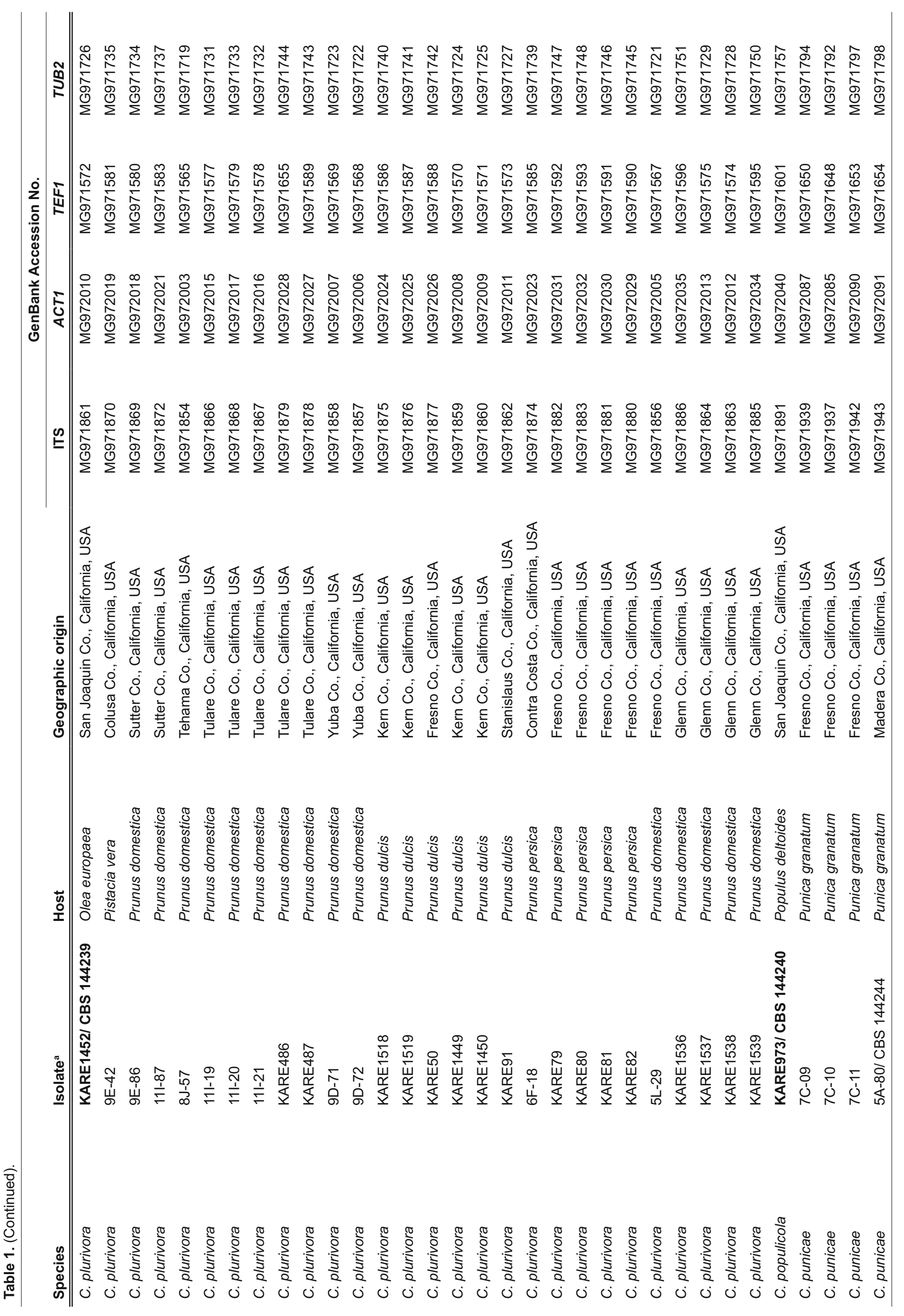




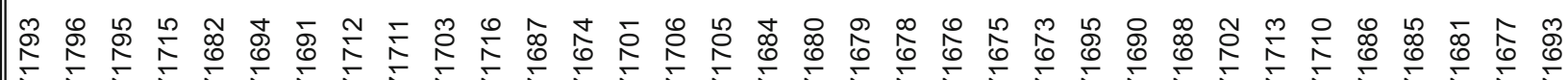

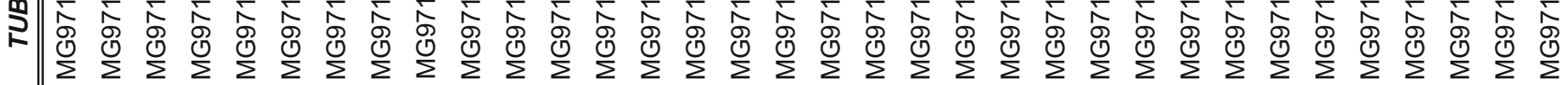

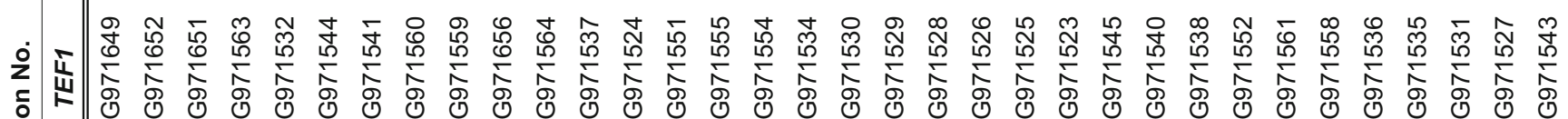

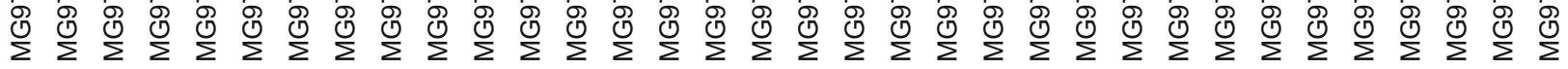

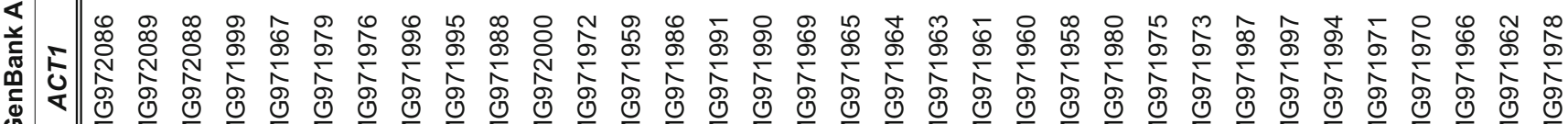

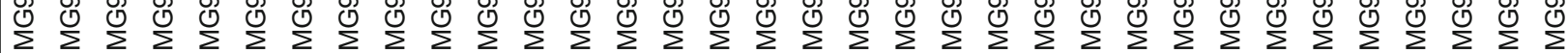

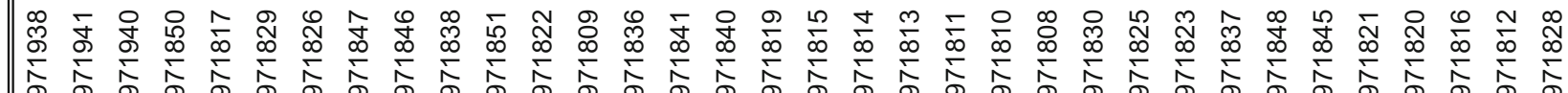

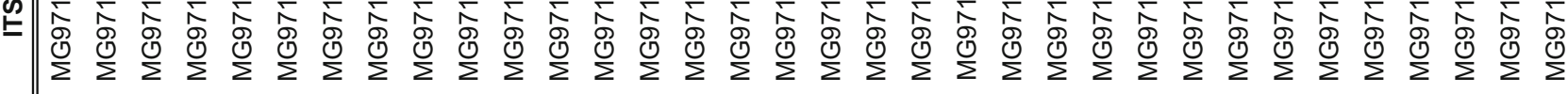
IIITII)

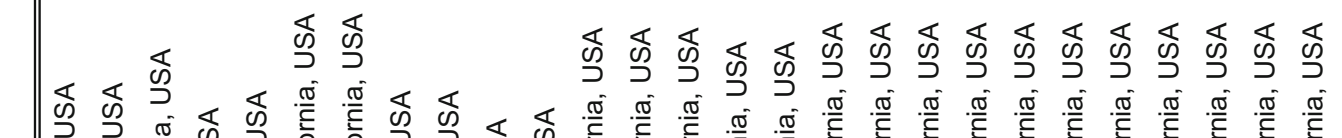

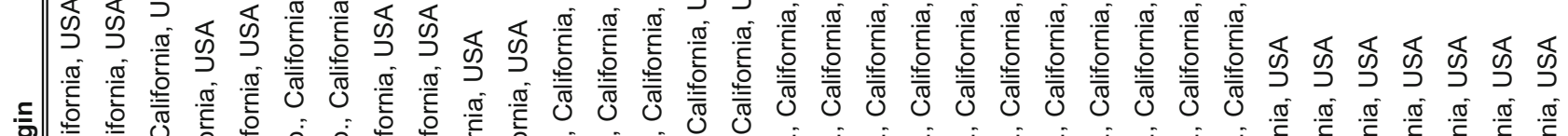

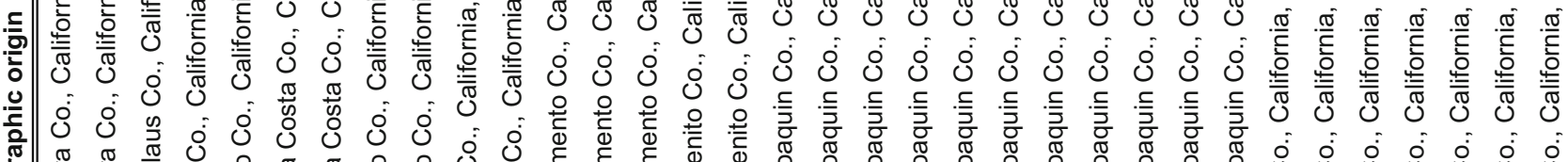

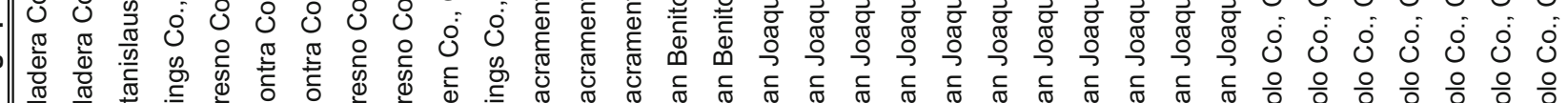

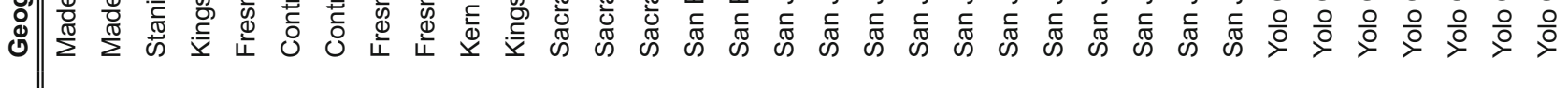



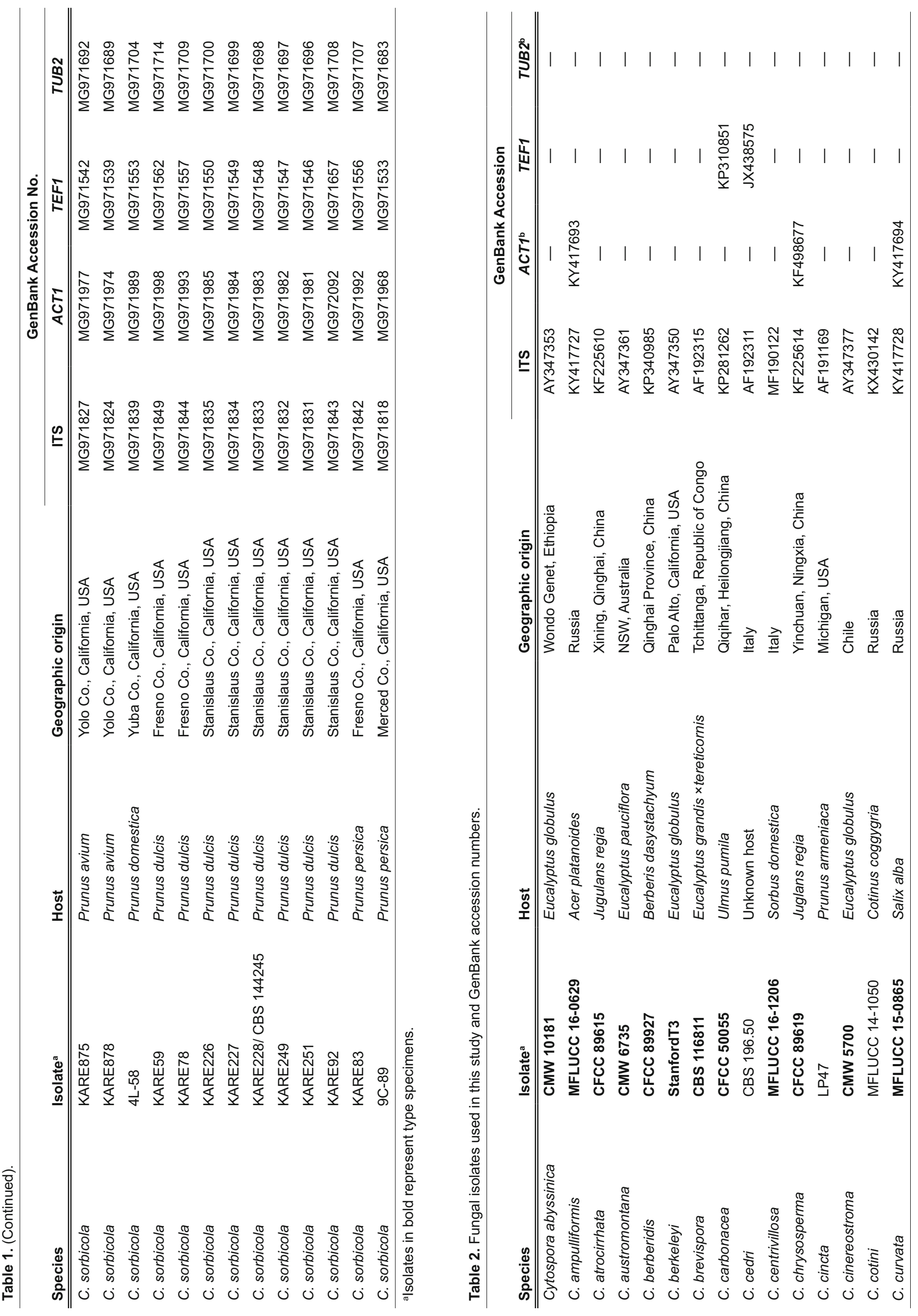


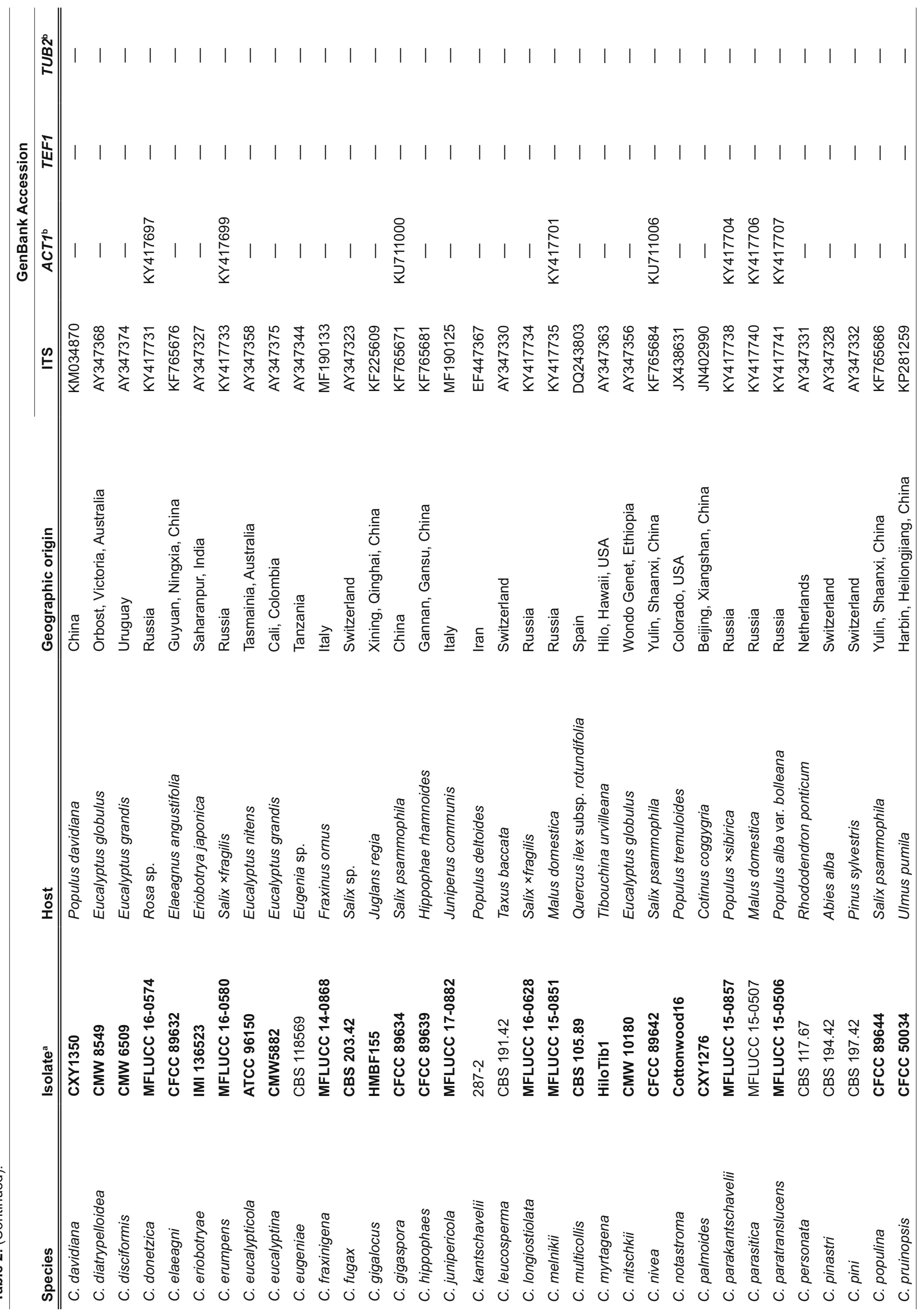




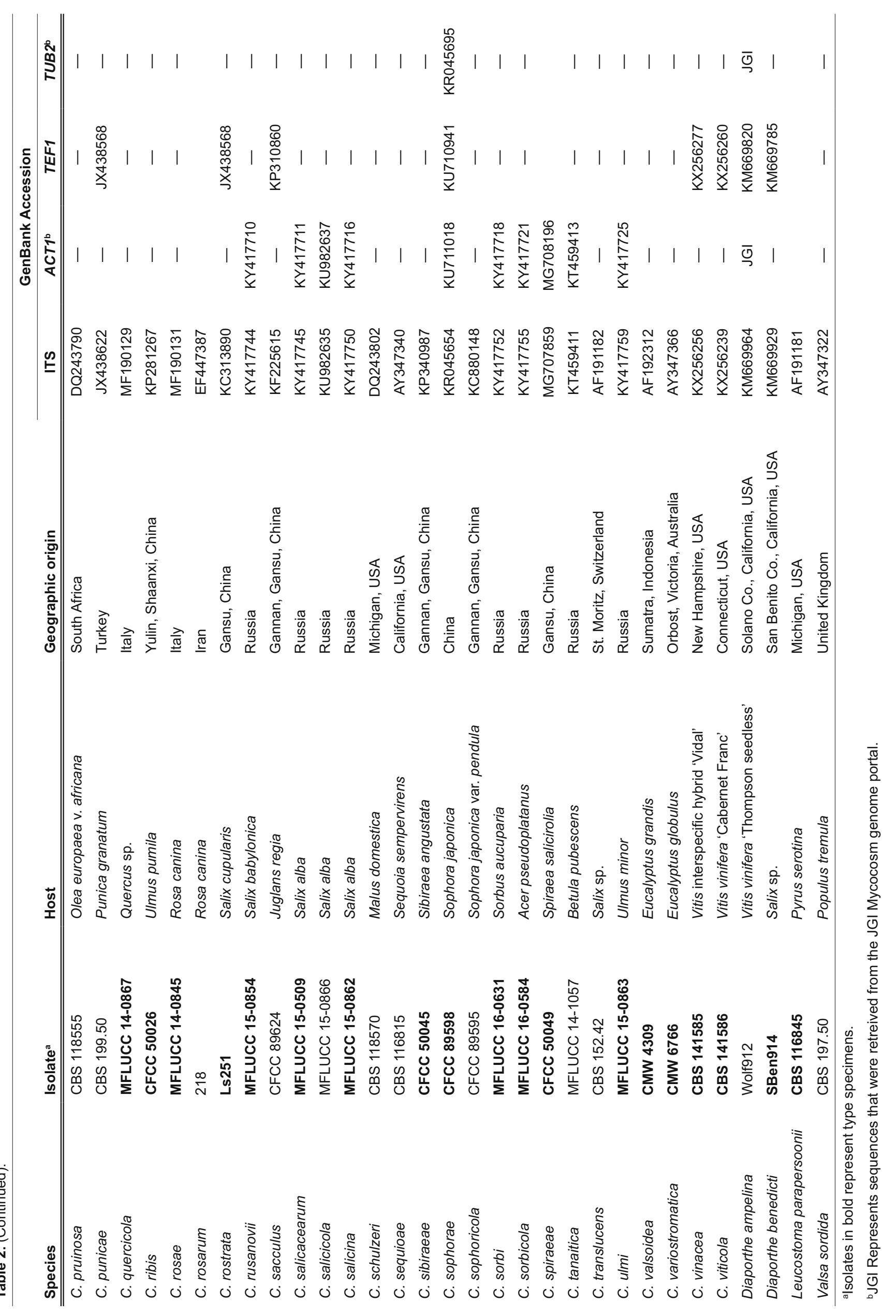


avium), olive (Olea europaea), peach (Prunus persica), pistachio (Pistacia vera), pomegranate (Punica granatum), prune (Prunus domestica), walnut (Juglans regia), and woody ornamentals such cottonwoods (Populus deltoides and $P$. fremontii), camellia (Camellia $\mathrm{sp}$.) and sequoia (Sequoiadendron giganteum). Cankers and accompanying branch and twig dieback were the most common symptoms associated with Cytospora species. Trees expressing Cytospora cankers were observed in Butte, Colusa, Contra Costa, Fresno, Glenn, Kern, Kings, Lake, Madera, Merced, Sacramento, San Benito, San Joaquin, Stanislaus, Sutter, Tehama, Tulare, Yolo, and Yuba counties in California. Dieback symptoms were most obvious during the warm summer months, although putative infections might have occurred during the rainy winter and early spring seasons in California. Symptoms of Cytospora canker includes bark lesions with dead phloem and cambium, discoloration of the xylem, wood necrosis and gumming occurring at the canker margin. Cankers were often depressed or sunken, eventually causing splitting of the bark or girdling of branches. Cankers were most commonly associated with pruning wounds, sunburn, and oil injuries. A single French prune orchard in Yuba County, where the grower re-planted trees to fill the gaps from trees killed by Cytospora canker, showed $92 \%$ Cytospora infection of pruning cuts made to select the main scaffolds of the newly planted trees. Wood cankers expressed as wedge shaped to irregularly shaped vascular discolorations of the xylem tissue below the affected bark area. Eventually, pycnidia occurred just beneath the periderm giving the bark a pimpled appearance diagnostic of Cytospora infection. Removing the periderm generally exposed numerous, solitary and scattered pycnidia. Erumpent pycnidia eventually ruptured the bark outermost layers exposing white (characteristic in branches of French prune) apical discs above the cankered area or in the dead branches and twigs. Spore tendrils consisting of conidial masses (cirrus) exuding from pycnidia generally were visible in the orchards following spring rains. Signs and symptoms of Cytospora associated cankers in various fruit and nut host plants are illustrated in Figs 1-3.

\section{Phylogenetic analyses}

For $\mathrm{ML}$ analyses, the best-fit model of nucleotide evolution was deduced based on the AIC $(\mathrm{K} 2+\mathrm{G}+\mathrm{I}$ for both $A C T 1$ and $T E F 1, H K Y+G$ for TUB2, and ITS and combined analyses both utilized $G T R+G+I)$. PCR amplification of the ITS region generated 497-527 bp fragments and the alignment of 229 ITS sequences resulted in a 604-character dataset (350 characters were constant, 74 characters were parsimonyuninformative, and 180 characters were parsimony informative $(30 \%))$. MP analyses produced a single most parsimonious tree of 973 steps and a consistency index $(\mathrm{Cl})$, retention index $(\mathrm{RI})$, and rescaled consistency index $(\mathrm{RC})$ of $0.4193,0.8813$, and 0.3692 , respectively. PCR amplification of the TEF1 locus generated 588-664 bp fragments and the alignment of 161 TEF1 sequences resulted in a 799-character dataset (313 characters were constant, 124 characters were parsimony-uninformative, and 362 characters were parsimony informative (45\%)). MP analyses produced four equally most parsimonious trees of 1411 steps and a $\mathrm{Cl}, \mathrm{RI}$, and $\mathrm{RC}$ of $0.5506,0.9470$, and 0.5227 , respectively. PCR amplification of the TUB2 locus was problematic for 14 isolates, which reside in sister clades as described below, nevertheless PCR amplification of the TUB2 locus generated 527-554 bp fragments and the alignment of 136 TUB2 sequences resulted in a 575-character dataset (428 characters were constant, 28 characters were parsimony-uninformative, and 119 characters were parsimony informative (21\%)). MP analyses produced four equally most parsimonious trees of 350 steps and a $\mathrm{Cl}, \mathrm{RI}$, and $\mathrm{RC}$ of $0.6171,0.9485$, and 0.5834 , respectively. PCR amplification of the ACT1 locus generated 280-298 bp fragments and the alignment of 184 ACT1 sequences resulted in a 365-character dataset (149 characters were constant, 74 characters were parsimonyuninformative, and 142 characters were parsimony informative (39 \%)). MP analyses produced a single most parsimonious tree of 585 steps and a Cl, RI, and RC of $0.4825,0.9308$, and 0.4836 , respectively. The analysis of individual datasets yielded similar trees that only differed in the order of species divergences and varying levels of clade support (ITS, Fig. S1; TEF1, Fig. S2; TUB2, Fig. S3; and ACT1, Fig. S4).

The multi-locus dataset consisted of 2334 characters (1242 characters were constant, 293 characters were parsimony-uninformative, and 799 characters were parsimony informative (34 \%)). MP analysis produced a single most parsimonious tree of 3434 steps and a $\mathrm{Cl}, \mathrm{RI}$, and $\mathrm{RC}$ of $0.4947,0.9253$, and 0.4589 , respectively. MP and $\mathrm{ML}$ analyses revealed that 150 Californian Cytospora isolates were divided into 15 species, five of which have been described previously (C. chrysosperma, C. parakantschavelli, C. punicae, C. sorbicola, and Valsa eucalypti) and 10 of which are not associated with a type or non-type isolate with DNA sequence data and thus represent novel phylogenetic species (Fig. 4). Descriptions of all species and taxonomic proposals are provided in the species descriptions and taxonomy section below.

\section{TAXONOMY}

Morphological comparisons coupled with multi-locus phylogenetic analyses (MP and ML) of the combined fourgene dataset identified 10 distinct and strongly supported lineages for which no apparent species names exist. Thus, we propose the following new species names and a new combination to properly circumscribe these unique taxa. Additionally, two previously described species are described from North America for the first time.

Fig. 4. The single most parsimonious tree generated from maximum parsimony analysis of the four-gene (ITS, TEF1, TUB2, and ACT1) combined dataset. Numbers in front and after the slash represent parsimony and likelihood bootstrap values from 1000 replicates, respectively. Values represented by an asterisk were less than $70 \%$ for the bootstrap analyses. Ex-type isolates are indicated in bold. Bar indicates the number of nucleotide changes. 
3G-09 Prunus avium CA, USA

KARE626 Prunus avium CA, USA

KARE 876 Prunus avium CA, USA

KARE879 Prunus avium CA, USA

KARE614 Prunus avium CA, USA

KARE618 Prunus avium CA, USA

KARE621 Prunus avium CA, USA

KARE624 Prunus avium CA, USA

KARE162 Prunus avium CA, USA

KARE92 Prunus avium CA, USA

KARE874 Prunus avium CA, USA

36/" KARE612 Prunus avium CA, USA

KARE615 Prunus avium CA, USA

KARE616 Prunus avium CA, USA

KARE619 Prunus avium CA, USA

KARE617 Prunus avium CA, USA

KARE613 Prunus avium CA, USA

KARE878 Prunus avium CA, USA

KARE875 Prunus avium CA, USA

KARE625 Prunus avium CA, USA

KARE623 Prunus avium CA, USA

KARE622 Prunus avium CA, USA

KARE590 Prunus avium CA, USA

OF. KARE589 PrunUs avium CA, USA

KARE1241 Prunus avium CA, USA

KARE877 Prunus avium CA, USA

KARE881 Prunus avium CA, USA

5D-44 Prunus avium CA, USA

5D-48 Prunus avium CA, USA

KARE882 Prunus avium CA, USA

KARE 1451 Olea europaea CA, USA

91/94 KARE83 Prunus persica CA, USA

KARE228/CBS 144245 Prunus dulcis CA, USA

4L-58 Prunus domestica CA, USA

KARE251 Prunus dulcis CA, USA

KARE227 Prunus dulcis CA, USA

KARE59 Prunus dulcis CA, USA

KARE249 Prunus dulcis CA, USA

9C-89 Prunus persica CA, USA

KARE226 Prunus dulcis CA, USA

MFLUCC 16-0584 Acer pseudoplatanus Russia

Cytospora sorbicola

Cytospora donetzica MFLUCC 16-0574 Rosa sp. Russia

Cytospora erumpens MFLUCC 16-0580 Salix xfragilis Russia

$99 /\left.99\right|_{\text {LH357/CBS } 144233 \text { Prunus du }} ^{\text {LH356 Prunus dulcis CA, USA }}$

Cytospora amygdali sp. nov.

$8 \mathrm{C}-55$ Juglans regia CA, USA

6F-18 prunus persica CA, USA

9F-03 Juglans regia CA, USA

74 9D-72 Prunus domestica CA, USA

9D-71 Prunus domestica CA, USA

97/78. KARE1539 Prunus domestica CA, USA

KARE1536 Prunus domestica CA, USA

-9F-01 Juglans regia CA, USA

111-87 prunus domestica CA, USA

111-87 Prunus domestica CA, USA

99/99 111-19 Prunus domestica CA, USA

92/99 111-20 Prunus domestica CA, USA

KARE1450 Prunus dulcis CA, USA

111-89 Juglans regia CA, USA

KARE1449 Prunus dulcis CA, USA

98/95 KARE1538 Prunus domestica CA, USA KARE1537 Prunus domestica CA, USA

Cytospora plurivora sp. nov.

5L-29 Prunus domestica CA, USA

96/96 KARE1452/CBS 144239 Olea europaea CA, USA

KARE91 Prunus dulcis CA, USA

- 8 J-57 Prunus domestica CA, USA

99/98 - 8J-57 Prunus domestica CA, USA

99/98 9 9E-86 Prunus domestica CA, USA

99/97 - 9E-42 Pistacia vera CA, USA

9F-08 Juglans regia CA, USA

KARE80 Prunus persica CA, USA

99/99- KARE1518 Prunus dulcis CA, USA

KARE1519 Prunus dulcis CA, USA

KARE487 Prunus domestica CA, USA

KARE486 Prunus domestica CA, USA

KARE50 Prunus dulcis CA, USA

KARE79 Prunus persica CA, USA

KARE82 Prunus persica CA, USA

98766 KARE81 Prunus persica CA, USA

- Cytospora sorbi MFLUCC 16-0631 Sorbus aucuparia Russia

Cytospora multicollis CBS 105.89 Quercus ilex subsp. rotundifolia Spain

Cytospora gigaspora CFCC 89634 Juglans regia China

Cytospora nivea CFCC 89642 Salix psammophila China

83/99 Cytospora translucens CBS 152.42 Salix sp. Switzerland

98/'- Cytospora paratrans/ucens MFLUCC 15-0506 Populus alba var. bolleana Russia

Cytospora loucostoma CBS 1166845 Prunus persica MI, USA

Cytospora davidiana CXY1350 Populus davidiana China

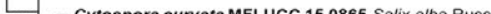

93/97 Cytospora rusanovii MFLUCC 15-0854 Salix babylonica Russia 
$\bigwedge \bigwedge$ Cyrospora pini CBS 197.42 Pinus sylvestris Switzerland

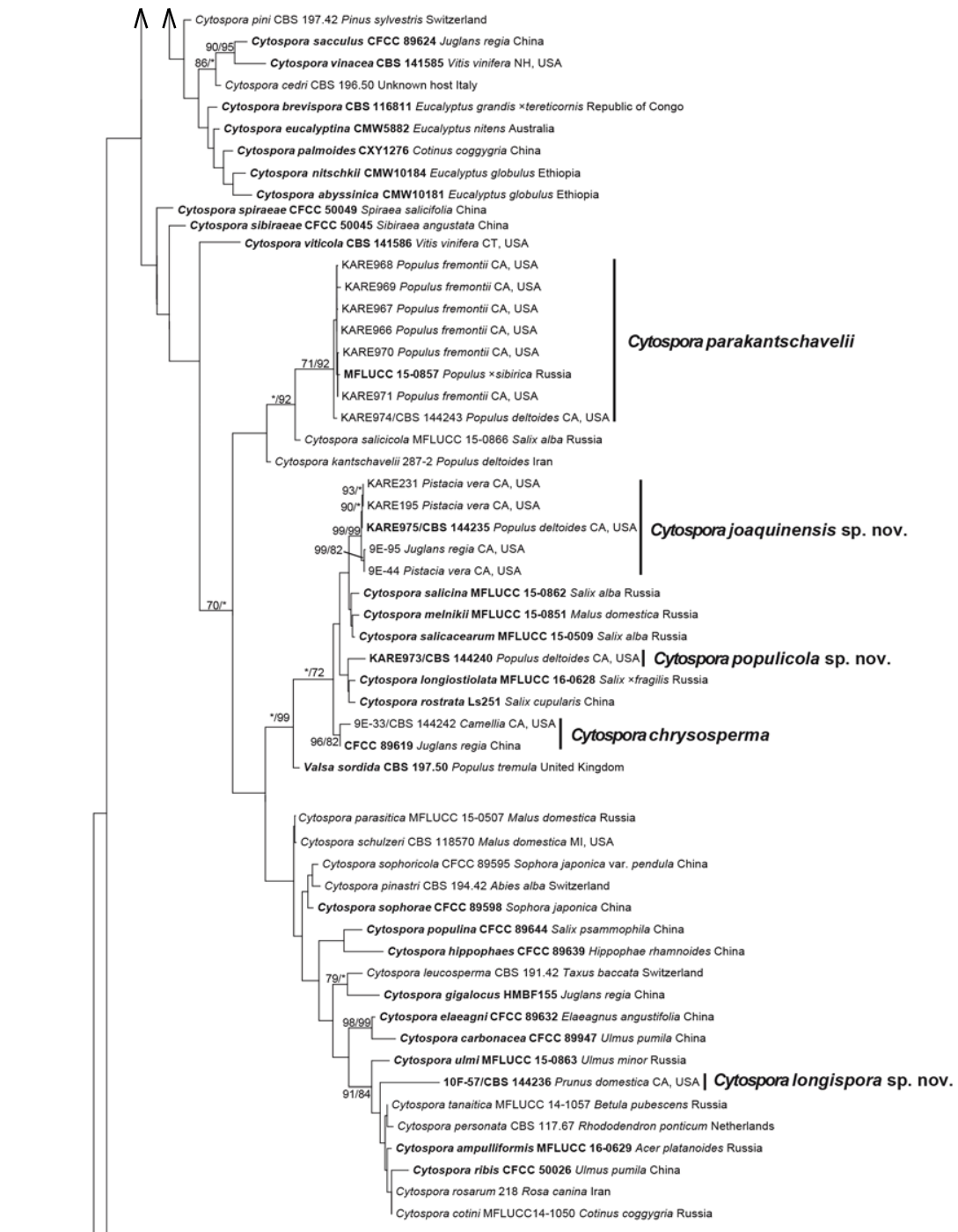

||

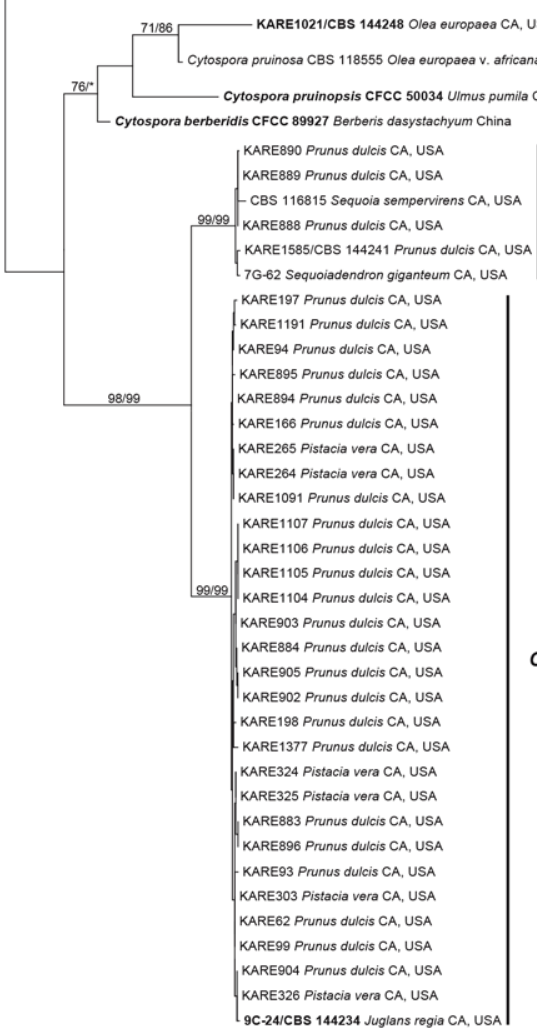

UA | Cytospora oleicola sp. nov.

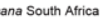

Cytospora eucalypti comb. nov.

Cytospora californica sp. nov.

Fig. 4. (Continued). 


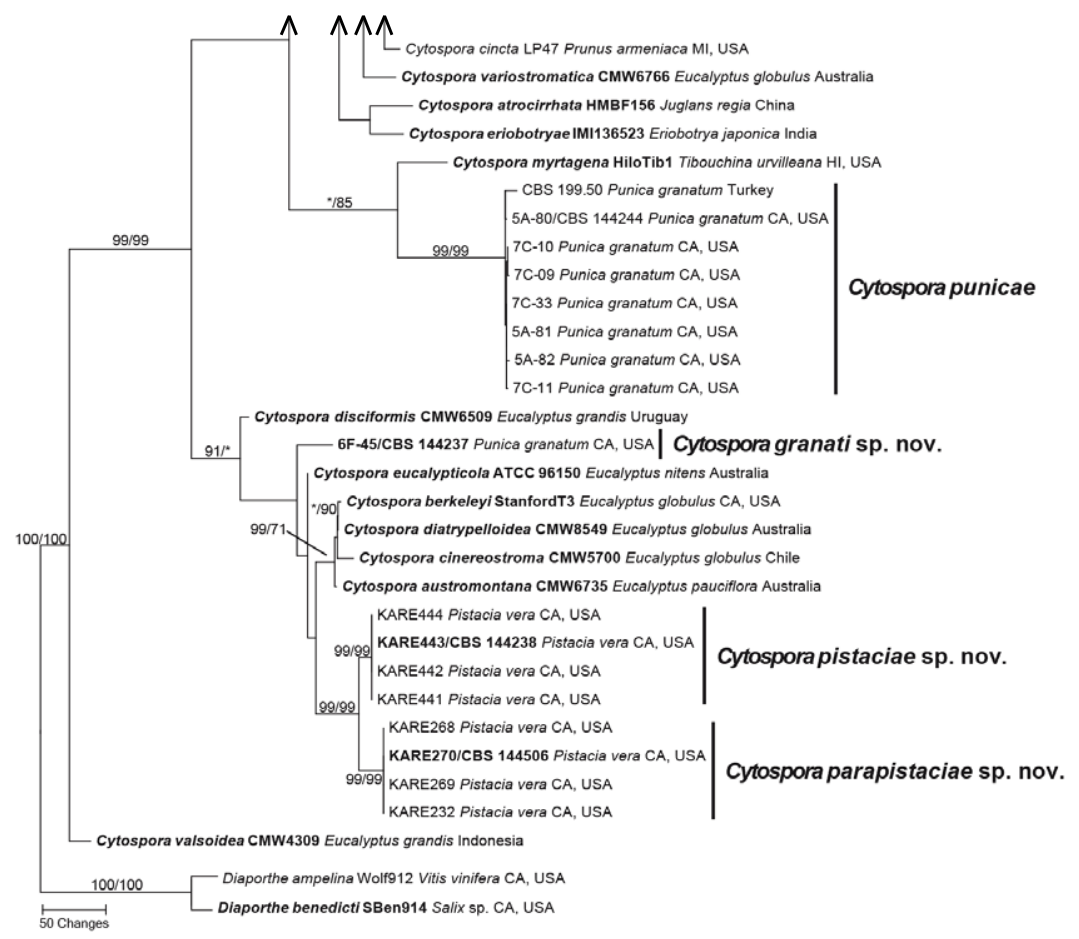

Fig. 4. (Continued).

Cytospora amygdali D.P. Lawr., L.A. Holland \& Trouillas, sp. nov. MycoBank MB824274 (Figs 4-5)

Etymology: The name refers to the host, almond.

Diagnosis: Cytospora amygdali can be distinguished from the phylogenetically closely related $C$. plurivora by the production of large robust conidia and solitary pycnidia in culture.

Type: USA: California: Yolo County, isolated from wood canker of Prunus dulcis, 3 Mar. 2016, L.A. Holland LH357 (BPI 910650 [dried culture] - holotype; CBS 144233 - exholotype culture).

Description: Conidiomata on PDA pycnidial, solitary, globose to subglobose, without conceptacle, mouse-grey in centre with white to off-white surface hyphae, (455-)570-690(-850) $\mu \mathrm{m}$ diam $(n=20)$, with $1-2$ internal locules. Conidiophores hyaline, smooth-walled, reduced to single monoblastic straight filamentous conidiogenous cells (5.5-)5.9-7.1(-7.5) $\times(1.0-) 0.9-1.1(-1.0) \mu \mathrm{m}(n=20)$, that are wider at the base and taper towards apex. Conidia abundant, relatively large with wide girth, single, hyaline, eguttulate, aseptate, allantoid, $(6.0-) 6.2-7.0(-7.0) \times(1.5-) 1.6-1.8(-2.0) \mu \mathrm{m}(n=30)$. No sexual morph observed.

Culture characteristics: Colonies after $7 \mathrm{~d}$ at $25^{\circ} \mathrm{C}$ on PDA average $57 \mathrm{~mm}$, medium-growing, slightly dentate, off-white outer margin, and cinnamon-colored inner margin with centre of the colony becoming dark mouse-grey with age. Hyphae hyaline, smooth, straight, branched, and septate.

Distribution: Yolo County (California, USA).
Host: Prunus dulcis.

Notes: Based on the phylogenetic inference obtained in this study, C. plurivora is the closest relative to $C$. amygdali, albeit without significant bootstrap support. Cytospora amygdali produces larger conidia, (6.0-)6.2-7.0(-7.0) × (1.5-)1.6$1.8(-2.0) \mu \mathrm{m}$, in terms of both length and width and pycnidia are always solitary, contrary to smaller conidia, (3.5-)3.8$4.4(-4.5) \times(1.0-) 0.9-1.1(-1.5) \mu \mathrm{m}$, and aggregated pycnidia produced by $C$. plurivora.

Cytospora californica D.P. Lawr., L.A. Holland \& Trouillas, sp. nov.

MycoBank MB824275

(Figs 4 and 6)

Etymology: The name refers to the geographical region, California, from where this fungus was first isolated.

Diagnosis: Cytospora californica can be distinguished from the species $C$. eucalypti by the former producing, on average, shorter conidia (C. californica (4.0-)4.5-5.5(-6.0) $\times(1.0-) 1.2-1.6(-1.5) \mu \mathrm{m}$ vs. C. eucalypti (5.0-)5.4-6.5($7.5) \times(1.0-) 1.2-1.6(-2.0) \mu \mathrm{m})$ and slower growth rate $(C$. californica $58.8 \mathrm{~mm}$ in $7 \mathrm{~d}$ vs. C. eucalypti $85 \mathrm{~mm}$ in $7 \mathrm{~d}$ ) and pattern in culture (C. californica produces two distinct margins in culture, with the internal margin darker than the peripheral margin, while C. eucalypti generally produces a homogenous pattern in culture).

Type: USA: California: Lake County, isolated from wood canker of Juglans regia, 14 Mar. 2014, T.J. Michailides 9C-24 (BPI 910651 [dried culture] - holotype; CBS 144234 - exholotype culture). 

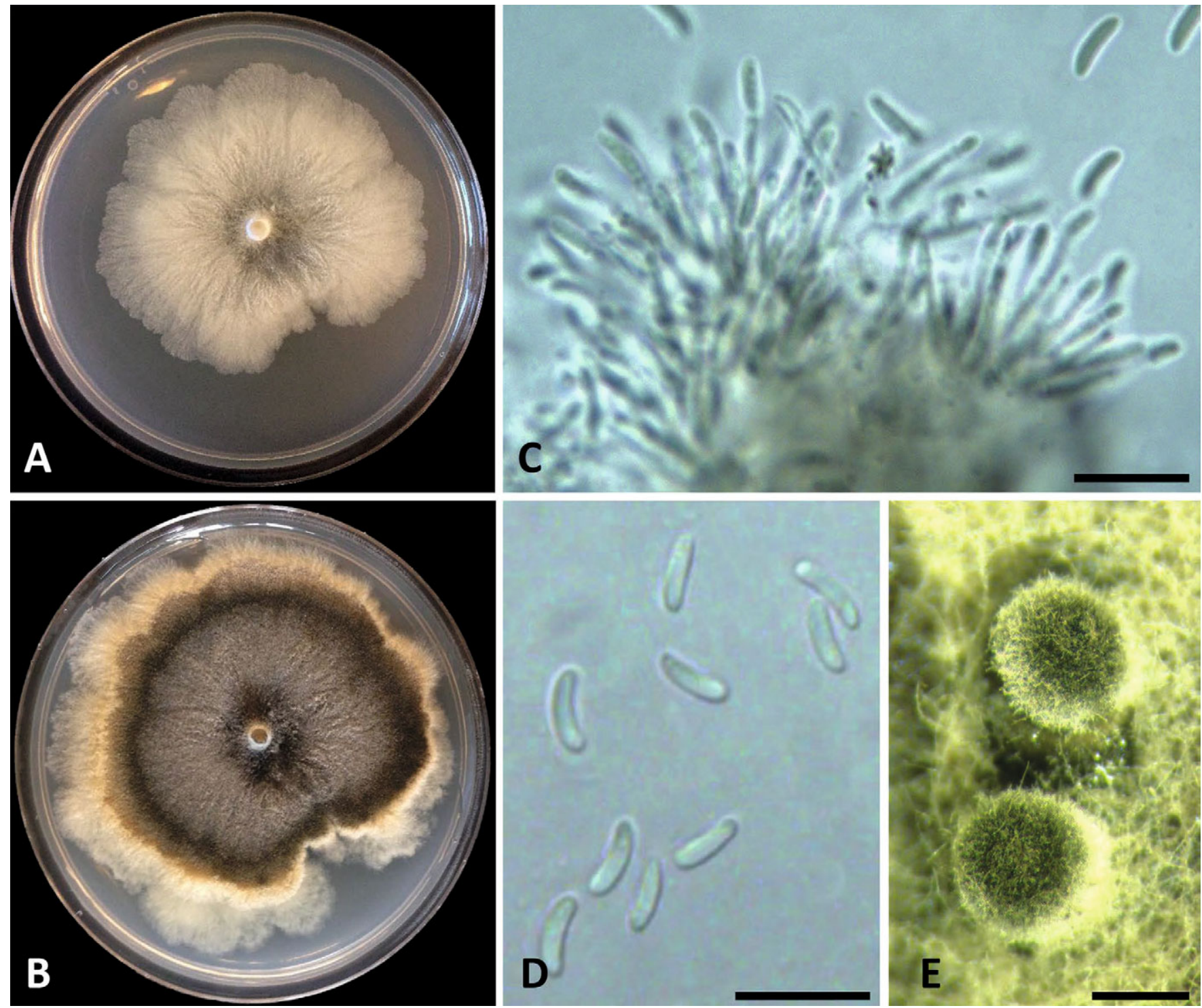

Fig. 5. Cytospora amygdali (ex-holotype culture CBS 144233). A. Seven-day-old PDA culture. B. Fourteen-day-old PDA culture. C. Conidiophores and filamentous conidiogenous cells. D. Conidia. E. Pycnidia. Bars C-D = $10 \mu \mathrm{m} ; \mathrm{E}=500 \mu \mathrm{m}$.

Description: Conidiomata on PDA pycnidial, mostly solitary, rarely aggregated, globose to subglobose, without conceptacle, white, (1255-)1356-1834(-2100) $\mu \mathrm{m}$ diam ( $n$ $=20$ ), with multiple internal locules with shared invaginated walls. Conidiophores hyaline, smooth-walled, reduced to 3-4 monoblastic branching filamentous conidiogenous cells $(5.0-) 5.9-7.9(-9.0) \times(1.0-) 1.1-1.5(-1.5) \mu \mathrm{m}(n$ $=20$ ) that taper towards the apex. Conidia abundant, single, hyaline to brown, eguttulate, aseptate, allantoid, $(4.0-) 4.5-5.5(-6.5) \times(1.0-) 1.2-1.6(-2.0) \mu \mathrm{m}(n=30)$. No sexual morph observed.

Culture characteristics: Colonies after $7 \mathrm{~d}$ at $25^{\circ} \mathrm{C}$ on PDA average $58.8 \mathrm{~mm}$, medium-growing, margin mostly smooth with some unevenness, with short aerial tufts giving a cottony appearance, margin white to off-white with buff centre. Hyphae hyaline, smooth, straight, branched, and septate.
Distribution: Glenn, Fresno, Kern, Lake, San Joaquin, and Stanislaus Counties (California, USA).

Hosts: Juglans regia, Pistacia vera, and Prunus dulcis.

Notes: Based on the phylogenetic inference obtained in this study, C. eucalypti (syn. Valsa eucalypti) is the closest relative to C. californica. Most micro-morphological observations between the two species overlap, however the colony growth rate of $C$. californica is much slower $(58.8 \mathrm{~mm}$ in $7 \mathrm{~d})$ than that of $C$. eucalypti ( $85 \mathrm{~mm}$ in $7 \mathrm{~d}$ ), and $C$. californica produces, on average, shorter conidia (4.0-)4.5-5.5(-6.5) than C. eucalypti (5.0-)5.4-6.5(-7.5). Amplification of the TUB2 locus using the primers $\mathrm{Bt} 1 \mathrm{a} / \mathrm{Bt} 1 \mathrm{~b}$ was problematic for this taxon. Several different annealing temperatures were attempted (annealing temperature ranging from $50-60{ }^{\circ} \mathrm{C}$ ) with marginal success as only 19 out of $30 \mathrm{C}$. californica isolates produced a reliable TUB2 PCR amplicon. 

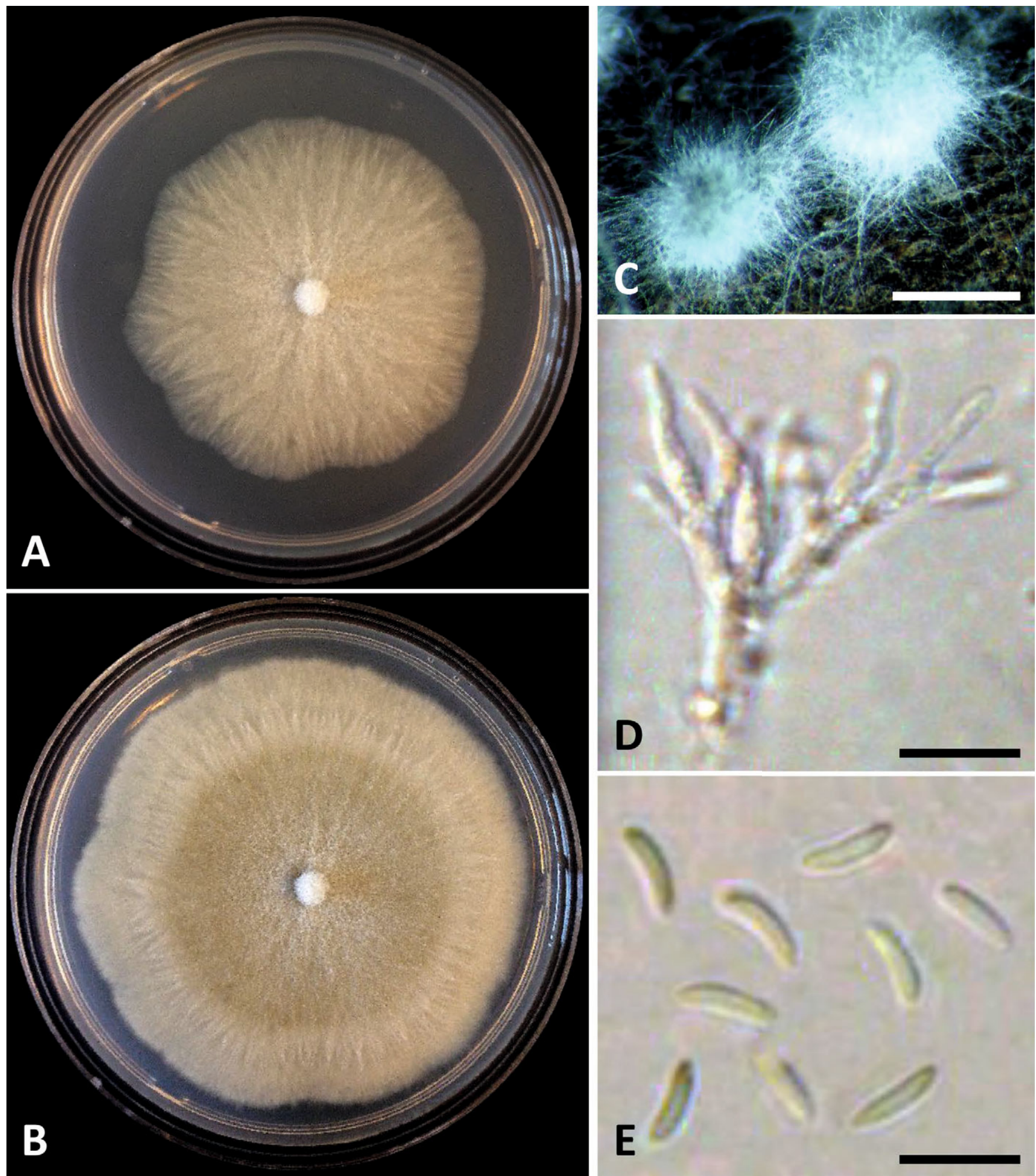

Fig. 6. Cytospora californica (ex-holotype culture CBS 144234). A. Seven-day-old PDA culture. B. Fourteen-day-old PDA culture. C. Pycnidia. D. Conidiophores and filamentous conidiogenous cells. E. Conidia. Bars $C=1 \mathrm{~mm} ; \mathrm{D}=10 \mu \mathrm{m} ; \mathrm{E}=5 \mu \mathrm{m}$.

Cytospora chrysosperma (Pers.) Fr., Syst. Mycol. 2(2): 542 (1823); nom. sanct.

Basionym: Sphaeria chrysosperma Pers., Neues Mag. Bot. 1: 82 (1794).

Synonyms: Naemaspora chrysosperma

(Pers.) Pers., Obs. Mycol. 1: 80 (1796). Naemaspora populina Spreng., Fl. Hal.: 354 (1806).

(Figs 4 and 7 )
Description: Conidiomata on PDA pycnidial, mostly solitary, sometimes aggregated, globose to subglobose, without conceptacle, grey with off-white surface hyphae, (960-)11191681 (-2070) $\mu \mathrm{m}$ diam $(n=20)$, with multiple internal locules with shared invaginated walls. Conidiophores some straight, some reduced to branching filamentous conidiogenous cells that taper towards the apex (7.0-)7.2-8.8(-10.0) $\times$ $(1.0-) 1.1-1.3(-1.5) \mu \mathrm{m}(n=20)$. Conidia abundant, single, 

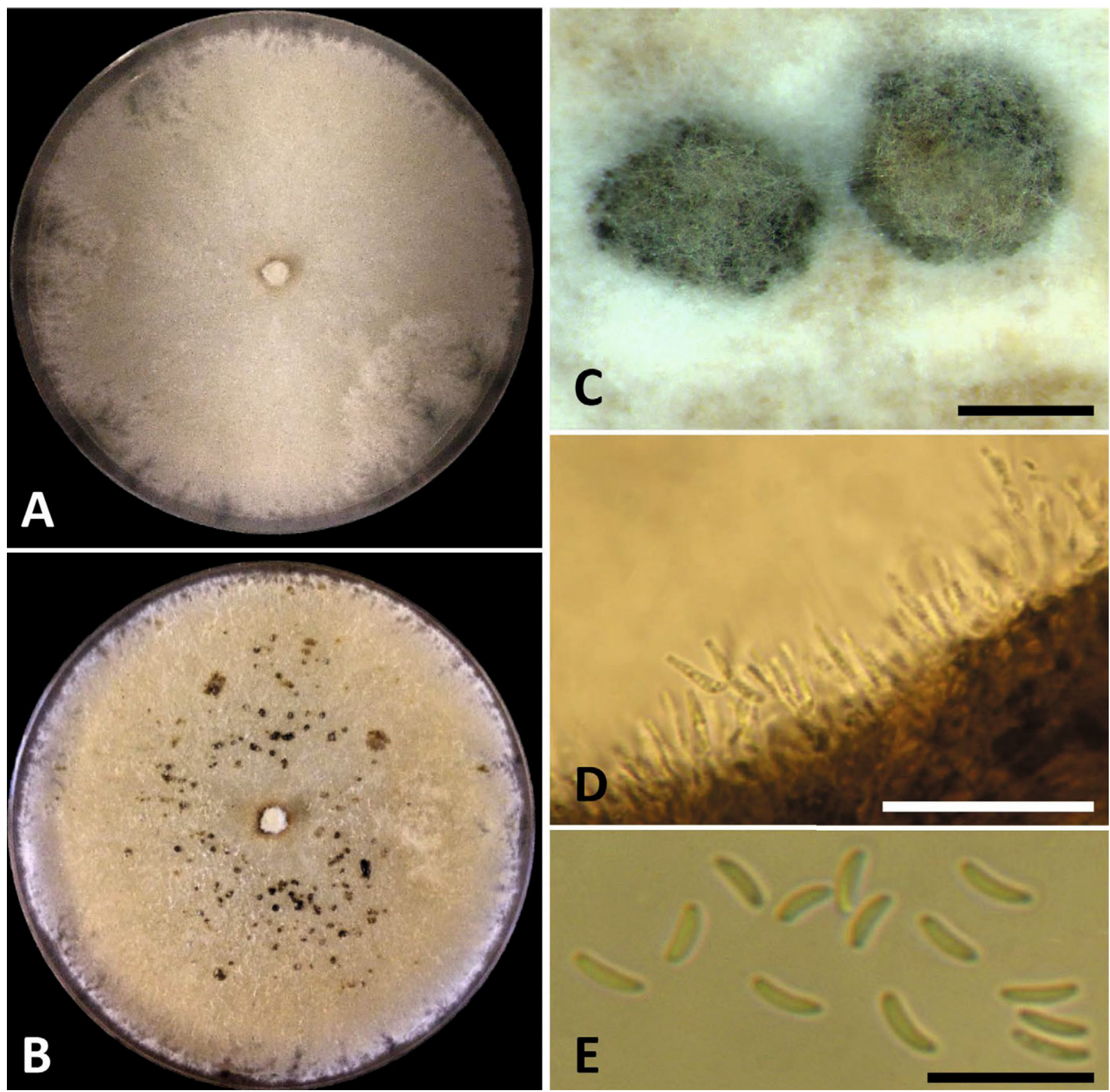

Fig. 7. Cytospora chrysosperma (CBS 144242). A. Seven-day-old PDA culture. B. Fourteen-day-old PDA culture. C. Pycnidia. D. Conidiophores and filamentous conidiogenous cells. E. Conidia. Bars $C=1 \mathrm{~mm} ; \mathrm{D}=20 \mu \mathrm{m} ; \mathrm{E}=10 \mu \mathrm{m}$.

hyaline to light brown, eguttulate, aseptate, allantoid, small, $(3.0-) 3.0-3.6(-4.0) \times(1.0-) 0.9-1.1(-1.0) \mu \mathrm{m}(n=30)$. No sexual morph observed.

Culture characteristics: Colony of C. chrysosperma isolate 9E-33, $90 \mathrm{~mm}$ diam in $7 \mathrm{~d}$ at $25^{\circ} \mathrm{C}$ on PDA, fast-growing, off-white to cream with short aerial tufts giving a cottony appearance, aerial hyphae becoming darker with age. Hyphae hyaline, smooth, straight, branched, and septate.

Distribution: China, Germany, Iran, The Netherlands, South Africa, Switzerland, the UK, and USA (Fresno County, California).
Hosts: The USDA Fungus-Host Distribution Database (https:// nt.ars-grin.gov/fungaldatabases/fungushost/fungushost. cfm lists more than 260 host records for C. chrysosperma, therefore a limited list is provided here: Crataegus azarolus, Ficus carica, Juglans regia, Ligustrum latifolium, Malus pumila, Morus alba, Olea sativa, Persica vulgaris, Prunus armeniaca, Prunus domestica, Robinia pseudoacacia, Salicaceae, Sophora japonica, Thuja orientalis, Triticum, Ulmus, and Vitis vinifera.

Notes: Based on the phylogenetic inference obtained in this study, C. chrysosperma is sister to the clade that contains C. joaquinensis, C. longiostiolata, C. melnikii, C. populicola, C. rostrata, C. salicacearum, and C. salicina. Cytospora chrysosperma is the type species of the genus, and CFCC 
89600 is an ex-type strain of the species (Fan et al. 2015) and our isolate $9 \mathrm{E}-33$ clusters strongly with that strain.

Specimen examined: USA, California: Fresno County, isolated from shoot of Camellia sp., 21 May 2014, T.J. Michailides 9E-33 (BPI 910652 [dried culture]; CBS 144242).

Cytospora eucalypti (Cooke \& Harkn.) D.P. Lawr., L.A. Holland \& Trouillas, comb. nov.

MycoBank MB824284

(Figs 4 and 8)

Basionym: Valsa eucalypti Cooke \& Harkn., Grevillea 9: 51 (1881).

Synonyms: Engizostoma eucalypti (Cooke \& Harkn.) Kuntze, Rev. Gen. Plant. 3(2): 474 (1884).

Valsa eucalypti var. myrti Rolland, Bull. Soc. Mycol. France

21: 22 (1905)

Leucostoma sequoiae Bonar, Mycologia 20: 295 (1928).

Type: USA: California: on dead branches of Eucalyptus globulus 1880, Cooke \& Harkness (UM 15128, MSC 11471 - isotypes).

Description: Conidiomata on PDA pycnidial, mostly solitary, rarely aggregated, globose, without conceptacle, dark black- grey, appearing dry, (990-)1268-1742(-2060) $\mu \mathrm{m}$ diam ( $=20$ ), with multiple internal locules with shared invaginated walls. Conidiophores short, reduced to branching filamentous conidiogenous cells tapering toward apices (5.5-)8.1-11.1($11.5) \times(1.0-) 1.3-2.1(-2.5) \mu \mathrm{m}(n=20)$. Conidia abundant, relatively large, single, hyaline, eguttulate, aseptate, allantoid, $(5.0-) 5.4-6.5(-7.5) \times(1.0-) 1.2-1.6(-2.0) \mu \mathrm{m}(n=50)$. No sexual morph observed.

Culture characteristics: Colonies after $7 \mathrm{~d}$ at $25^{\circ} \mathrm{C}$ on PDA average $85 \mathrm{~mm}$, fast-growing, buff to honey with short aerial tufts giving a cottony appearance, aerial hyphae becoming darker with age. Hyphae hyaline, smooth, straight, branched, and septate.

Distribution: Fresno, Marin, Merced, San Joaquin, and Santa Clara Counties (California, USA).

Hosts: Eucalyptus globulus, Eucalyptus paniculata, Eucalyptus sp., Prunus dulcis, Sequoia sempervirens, and Sequoiadendron gigateum.

Notes: The species name Cytospora eucalypti has been applied in the past (Sharma et al. 1985), however no type was indicated and this appeared in a research report that
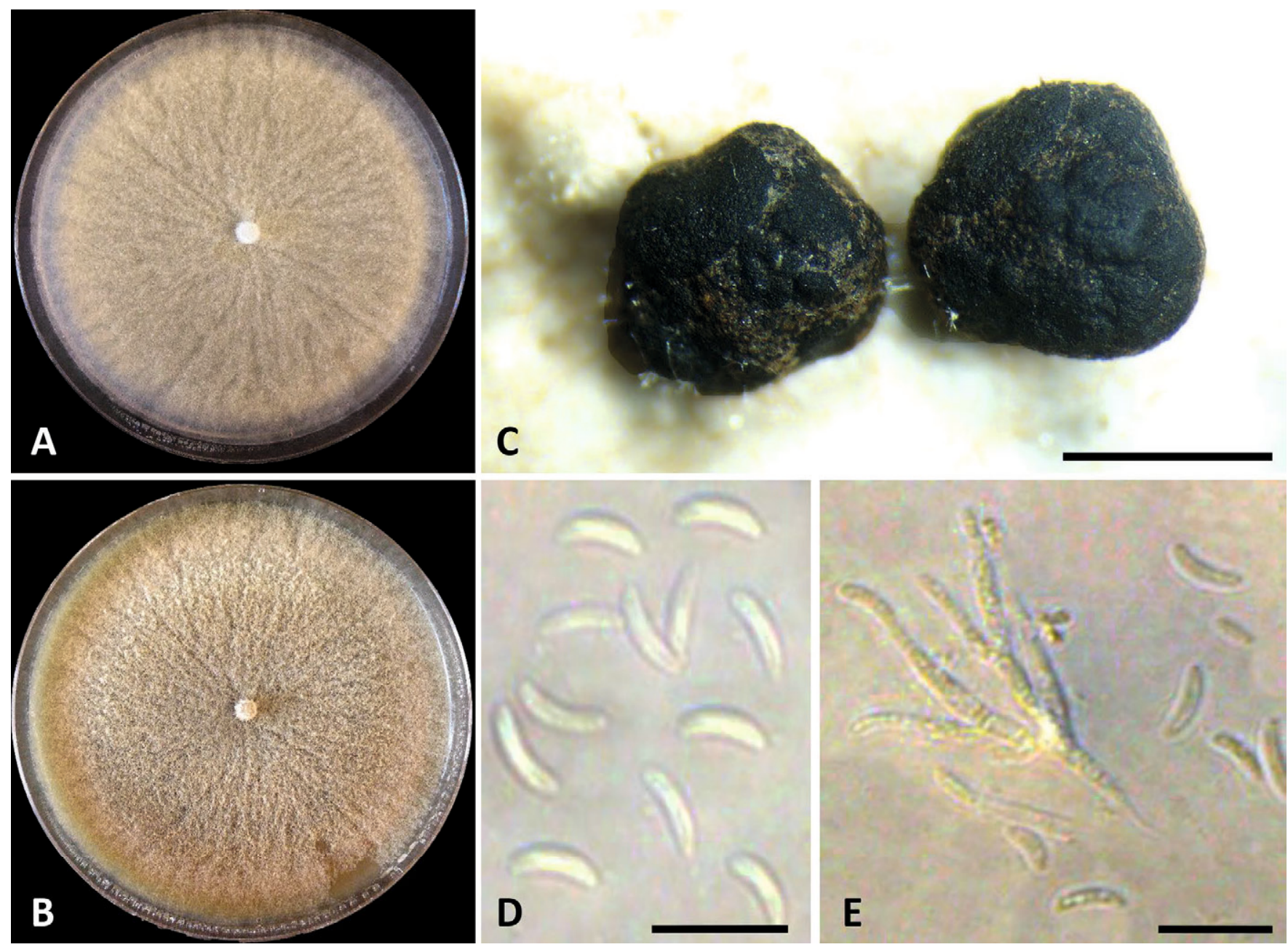

Fig. 8. Cytospora eucalypti (CBS 144241). A. Seven-day-old PDA culture. B. Fourteen-day-old PDA culture. C. Pycnidia. D. Conidia. E. Conidiophores and filamentous conidiogenous cells. Bars $C=1 \mathrm{~mm}$; $D-E=10 \mu \mathrm{m}$. 
was not effectively published, so the name was not validly published (Adams et al. 2005). The Californian isolates cluster strongly with an isolate named as Valsa eucalypti (CBS 116815 from Sequoia sempervirens) which also clusters with isolates collected from Eucalyptus in California (Adams et al. 2005, 2006). Based on the phylogenetic inference obtained in this study, C. eucalypti is sister to C. californica. Most morphological observations between the two species overlap, however, the colony growth rate of $C$. eucalypti is much faster $(85 \mathrm{~mm}$ in $7 \mathrm{~d}$ ) than that of C. californica (58.8 $\mathrm{mm}$ in $7 \mathrm{~d}$ ), and C. eucalypti produces, on average, longer conidia than C. californica (4.0-)4.5$5.5(-6.0)$. Amplification of the TUB2 locus using the primers $\mathrm{Bt} 1 \mathrm{a} / \mathrm{Bt} 1 \mathrm{~b}$ was problematic. Several different annealing temperatures were attempted with little success as only one out of five isolates produced a reliable TUB2 amplicon. Similar TUB2 PCR failures were encountered with the sister species $C$. californica, suggesting apomorphic nucleotide substitution(s) in these primer site(s).
Specimen examined: USA: California: Merced County, isolated from wood canker of Prunus dulcis, 28 Sep. 2016, F.P. Trouillas KARE1585 (BPI 910653 [dried culture]; CBS 144241).

Cytospora granati D.P. Lawr., L.A. Holland \& Trouillas, sp. nov.

MycoBank MB824278

(Figs 4 and 9)

Etymology: The name refers to the host, Punica granatum, from which this fungus was first isolated.

Diagnosis: Cytospora granati can be distinguished from $C$. eucalypticola by the former producing, on average, longer and wider conidia.

Type: USA: California: Tulare County, isolated from wood canker of Punica granatum, 29 Jul. 2011, T.J. Michailides 6F45 (BPI 910654 [dried culture] - holotype; CBS 144237 - exholotype culture).
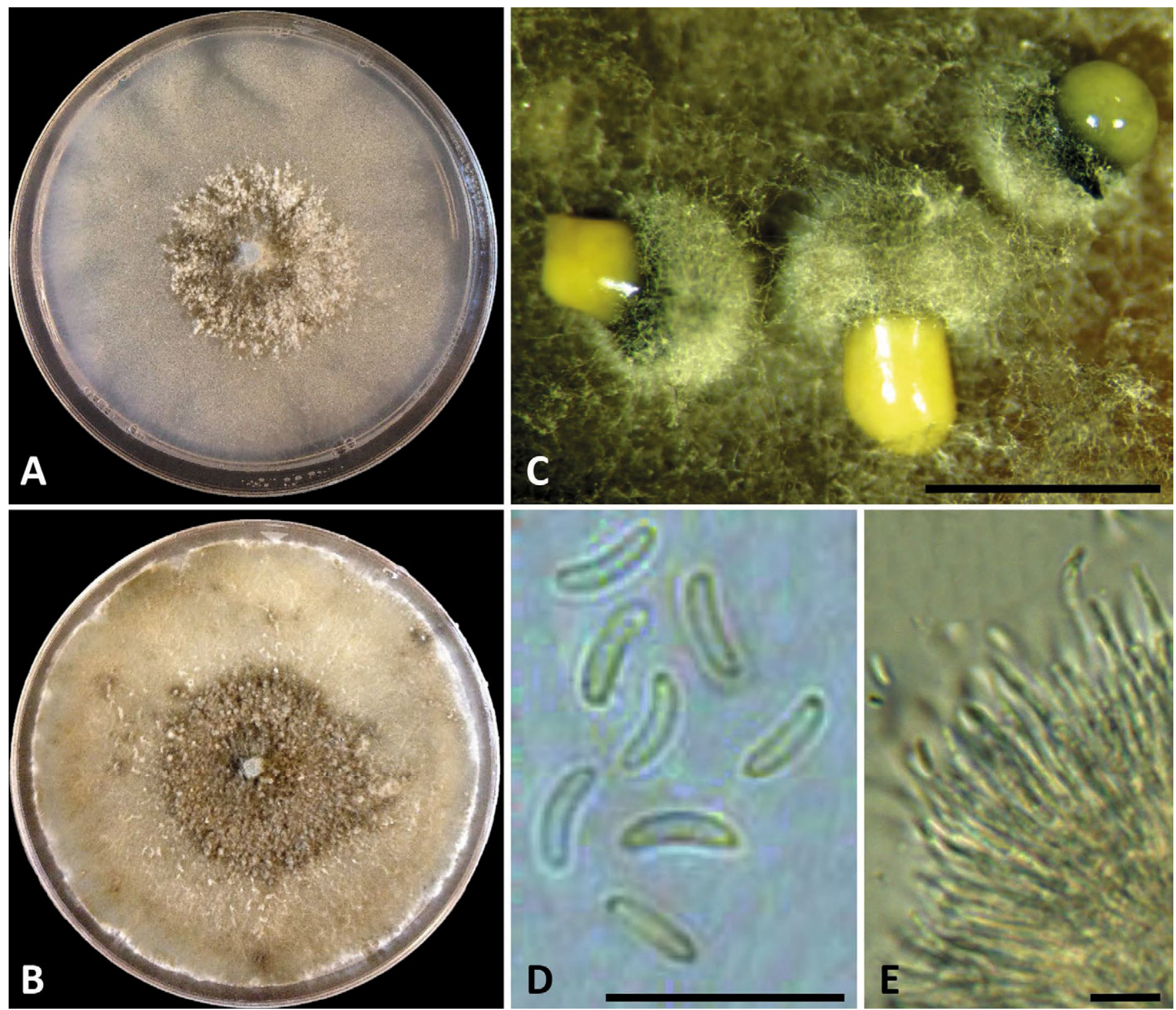

Fig. 9. Cytospora granati (ex-holotype culture CBS 144237). A. Seven-day-old PDA culture. B. Fourteen-day-old PDA culture. C. Pycnidia. D. Conidia. E. Conidiophores and filamentous conidiogenous cells. Bars $C=1 \mathrm{~mm} ; \mathrm{D}-\mathrm{E}=10 \mu \mathrm{m}$. 
Description: Conidiomata on PDA pycnidial, mostly solitary, sometimes aggregated, globose, conical to discoid, with yellow-coloured conidial exudate, without conceptacle, offwhite to light-grey, (610-)673-897(-975) $\mu \mathrm{m}$ diam $(n=20)$, with a single internal locule. Conidiophores reduced to straight filamentous conidiogenous cells (16.0-)19.3-23.5(-26.5) $\times(2.0-) 3.7-4.1(-5.0) \mu \mathrm{m}(n=20)$. Conidia copious, single, hyaline to light brown, aseptate, allantoid (4.0-)4.1-4.5(-5.0) $\times(1.0-) 1.1-1.3(-1.5) \mu \mathrm{m}(n=30)$. No sexual morph observed.

Culture characteristics: Colonies after $7 \mathrm{~d}$ at $25^{\circ} \mathrm{C}$ on PDA average $87.3 \mathrm{~mm}$, fast-growing, white to buff, raised mixed olivaceous white colony centre with flat colony expansion throughout with buff margin in mature colonies. Hyphae hyaline, smooth, straight, branched, and septate.

\section{Distribution: Tulare County (California, USA).}

Host: Punica granatum.

Notes: Based on the phylogenetic inference obtained in this study, C. granati resides in a clade that contains Cytospora species isolated from Eucalyptus in Australia (C. austromontana, C. diatrypelloidea, and C. eucalypticola), California (C. berkeleyi), Chile (C. cinereostroma), and Uruguay (C. disciformis), and from Pistacia vera in California (C. parapistaciae and C. pistaciae). This study identified two distantly related Cytospora species recovered from symptomatic pomegranate trees. Cytospora granati is easily distinguished from $C$. punicae by differences in pycnidial sizes (C. granati pycnidia (610-)673-897(-975) $\mu \mathrm{m}$ are almost twice as large, on average, as compared to $C$. punicae $(210-) 237-383(-490) \mu \mathrm{m})$ ), the much faster colony growth rate (C. granati $(87.3 \mathrm{~mm}$ in $7 \mathrm{~d})$ than $C$. punicae $(64.7 \mathrm{~mm}$ in $7 \mathrm{~d})$ ), and colony colour/morphology (C. granati produces a white to buff colony while $C$. punicae produces a characteristic dark red colony).

Cytospora joaquinensis D.P. Lawr., L.A. Holland \& Trouillas, sp. nov.

MycoBank MB824276

(Figs 4 and 10)

Etymology: The name refers to the San Joaquin Valley of California where the species was found.

Diagnosis: Cytospora joaquinensis can be distinguished from the related C. melnikii, C. salicacearum, and $C$. salicina as $C$. joaquinensis produces, on average, longer conidia.

Type: USA: California: San Joaquin County, isolated from wood canker of Populus deltoides, 21 Apr. 2016, F.P. Trouillas KARE975 (BPI 910655 [dried culture] - holotype; CBS 144235 - ex-holotype culture).

Description: Conidiomata on PDA pycnidial, mostly solitary, rarely aggregated, most with yellow conidial exudate, globose, no conceptacle, black-grey with off-white surface hyphae, (970-)1097-1533(-1760) $\mu \mathrm{m}$ diam ( $n=20)$, with multiple internal locules with shared invaginated walls. Conidiophores reduced to mostly straight unbranched filamentous conidiogenous cells (6.5-)7.7-10.1(-13.5) × (1.0-)1.1-1.3(1.5) $\mu \mathrm{m}(n=20)$. Conidia abundant, single, hyaline to light brown, eguttulate, aseptate, allantoid, (5.0-)5.1-5.7(-6.0) $\times$ (1.0-)1.1-1.3(-1.5) $\mu \mathrm{m}(n=30)$. No sexual morph observed.

Culture characteristics: Colonies after $7 \mathrm{~d}$ at $25^{\circ} \mathrm{C}$ on PDA average $86.7 \mathrm{~mm}$, fast-growing, buff-coloured with short aerial tufts giving a cottony appearance, aerial hyphae becoming darker with age, centre becoming honey-coloured that extends to a white margin. Hyphae hyaline, smooth, straight, branched, and septate.

Distribution: Fresno, Kern, San Joaquin, and Tulare Counties (California, USA).

Hosts: Juglans regia, Pistacia vera, and Populus deltoides.

Notes: Based on the phylogenetic inference obtained in this study, C. melnikii, C. salicacearum, and C. salicina are the closest relatives to $C$. joaquinensis. Conidia of $C$. joaquinensis $(5.0-) 5.1-5.7(-6.0) \times(1.0-) 1.1-1.3(-1.5)$, on average, are longer than $C$. melnikii $(3.1-) 4.5-5 \times 1-1.2(-1.3) \mu \mathrm{m}, C$. salicacearum (3.6-)4.9-6.4 × 0.9-1(-1.3) $\mu \mathrm{m}$, and C. salicina (3.6-)4.2-4.7 × 1-1.1(-1.3) $\mu \mathrm{m}$ (Norphanphoun et al. 2017).

Cytospora longispora D.P. Lawr., L.A. Holland \& Trouillas, sp. nov.

MycoBank MB824277

(Figs 4 and 11)

Etymology: The name refers to the exceptionally long conidia of this species.

Diagnosis: Unique mosaic colony morphology and conidia that are relatively long (6.0-)6.6-7.4(-7.5) × (1.0-)1.1-1.4(1.5) $\mu \mathrm{m}$ as compared to most other Cytospora species.

Type: USA: California: Glenn County, isolated from wood canker of Prunus domestica, 22 Oct. 2014, T.J. Michailides 10F-57 (BPI 910656 [dried culture] - holotype; CBS 144236 - ex-holotype culture).

Description: Conidiomata on PDA pycnidial, solitary, sometimes aggregated, many with cream-coloured conidial exudate, globose, no conceptacle, (805-)827-1393(-1635) $\mu \mathrm{m}(n=20)$, with a single internal locule. Conidiophores smooth-walled, straight, reduced to filamentous conidiogenous cells $(6.5-) 7.9-10.9(-11.5) \times(1.0-) 1.0-1.4(-$ 1.5) $\mu \mathrm{m}(n=20)$. Conidia long, abundant, single, hyaline, eguttulate, aseptate, allantoid, (6.0-)6.6-7.4(-7.5) $\times(1.0-)$ $1.1-1.4(-1.5) \mu \mathrm{m}(n=30)$. No sexual morph observed.

Culture characteristics: Colonies after $7 \mathrm{~d}$ at $25^{\circ} \mathrm{C}$ on PDA average $67.3 \mathrm{~mm}$, medium-growing, white to buff with short aerial tufts giving a cottony appearance in the centre, radially growing hyphae submerged, hyphae becoming darker with age. Outer margin a mosaic of sienna and amber with dark patches and a buff margin. Hyphae hyaline, smooth, straight, branched, and septate. 

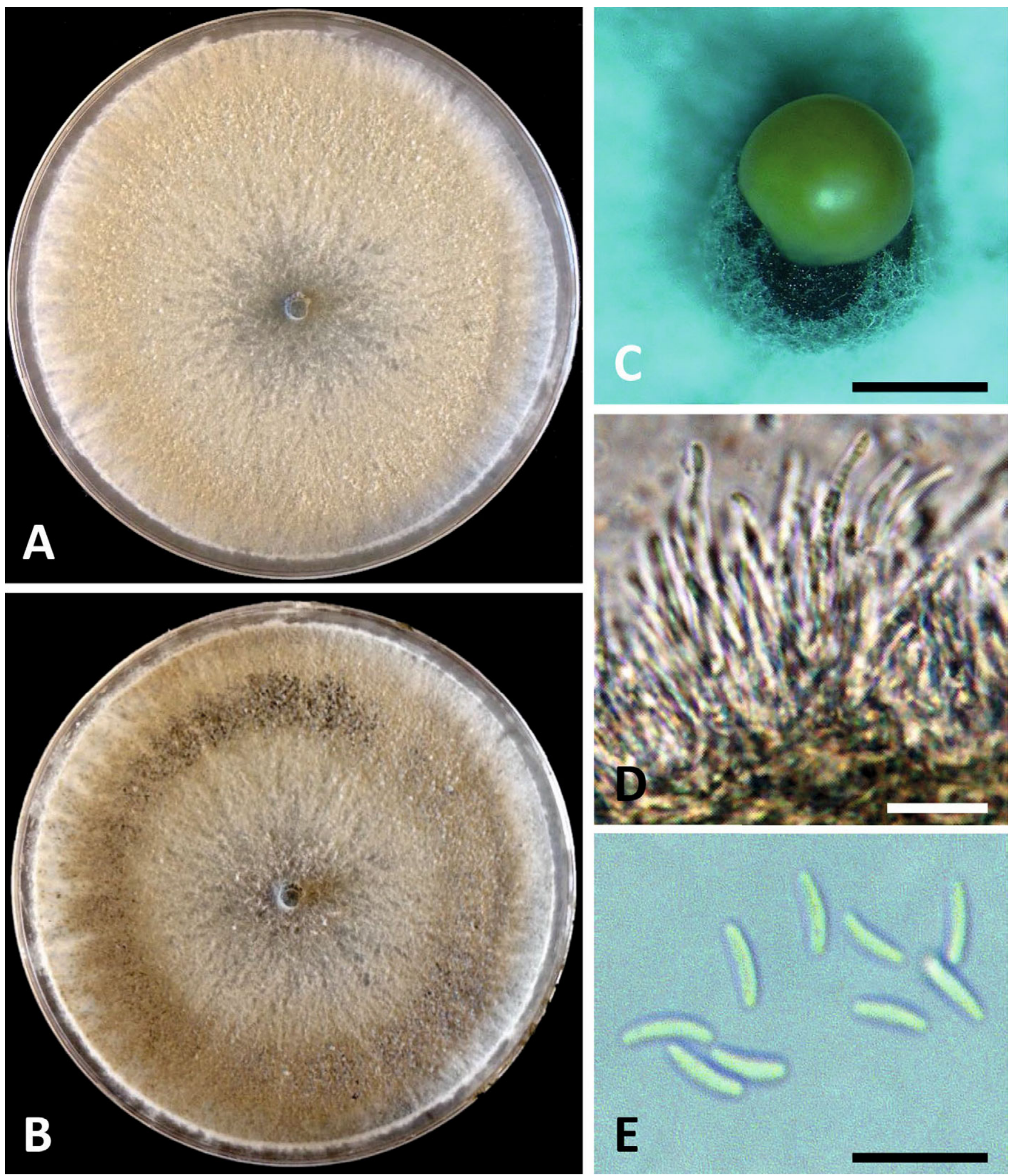

Fig. 10. Cytospora joaquinensis (ex-holotype culture CBS 144235). A. Seven-day-old PDA culture. B. Fourteen-day-old PDA culture. C. Pycnidium. D. Conidiophores and filamentous conidiogenous cells. E. Conidia. Bars $C=1 \mathrm{~mm} ; \mathrm{D}=20 \mu \mathrm{m} ; \mathrm{E}=10 \mu \mathrm{m}$.

Distribution: Glenn County (California, USA).

\section{Host: Prunus domestica.}

Notes: Based on the phylogenetic inference obtained in this study, C. longispora clusters in a strongly supported clade that contains C. ampulliformis, C. cotini, C. personata, C. ribis, C. rosarum, C. tanaitica, and C. ulmi. Conidia of all relatives are, on average, much shorter than $C$. longispora, with the exception of the recently described $C$. ampulliformis which produces larger conidia to $9 \mu \mathrm{m}$ in length (Norphanphoun et al. 2017). 

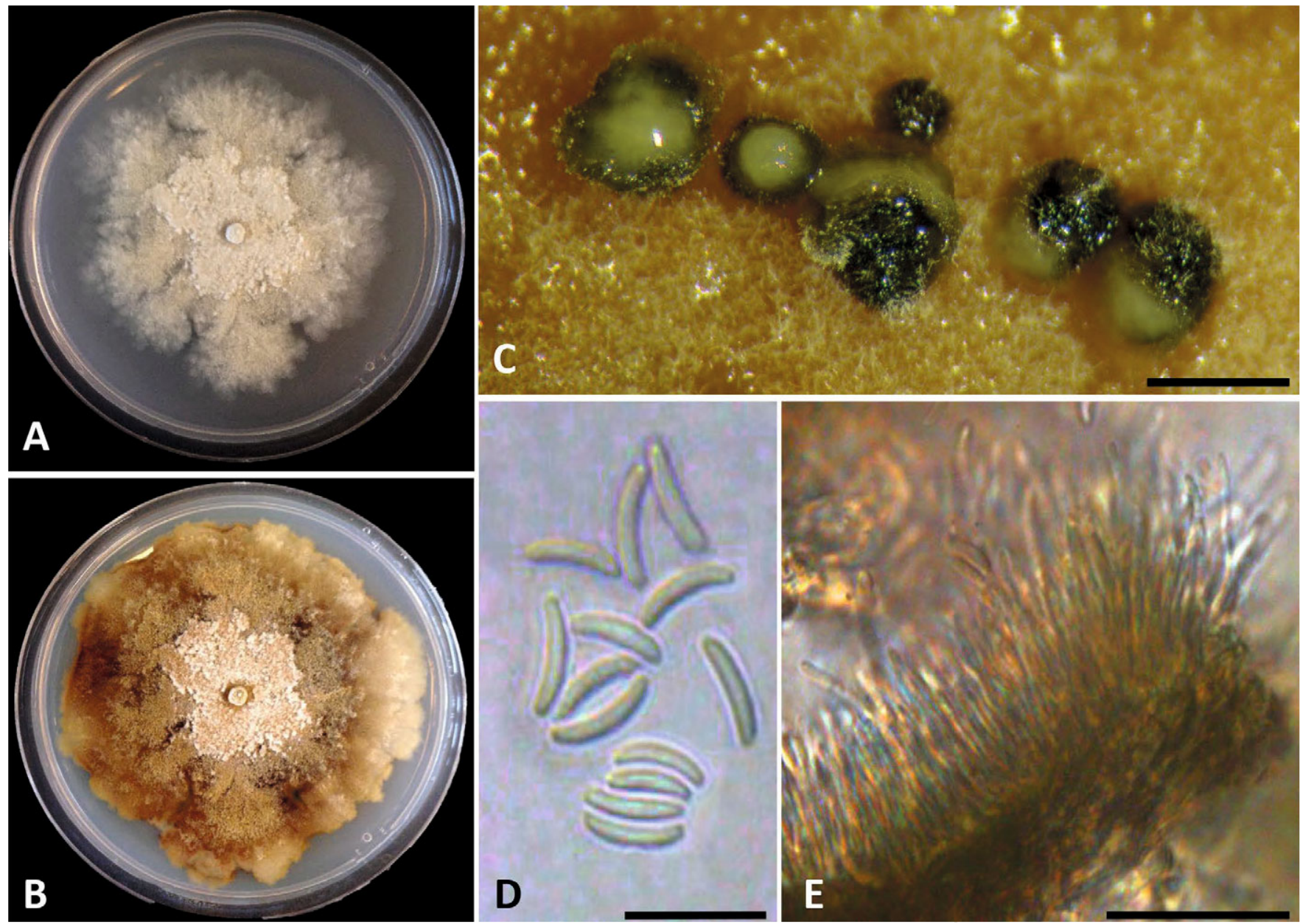

Fig. 11. Cytospora longispora (ex-holotype culture CBS 144236). A. Seven-day-old PDA culture. B. Fourteen-day-old PDA culture. C. Pycnidia.

D. Conidia. E. Conidiophores and filamentous conidiogenous cells. Bars $C=1 \mathrm{~mm} ; \mathrm{D}=10 \mu \mathrm{m} ; \mathrm{E}=20 \mu \mathrm{m}$.

Cytospora oleicola D.P. Lawr., L.A. Holland \& Trouillas, sp. nov.

MycoBank MB824279

(Figs 4 and 12)

Etymology: The name refers to the host Olea and -cola for inhabitor.

Diagnosis: Conidia of $C$. oleicola are wider and longer, on average, as compared to the closely related C. pruinosa.

Type: USA: California: San Joaquin County, isolated from twig canker of Olea europaea, 19 Apr. 2016, F.P. Trouillas KARE1021 (BPI 910657 [dried culture] - holotype; CBS 144248 - ex-holotype culture).

Description: Conidiomata on PDA pycnidial, mostly solitary, rarely aggregated, globose, light mouse-grey to almost black (640-)715-1185(-1545) $\mu \mathrm{m}$ diam $(n=20)$, with a single internal locule. Conidiophores straight, reduced to branching filamentous conidiogenous cells $(6.5-) 7.5-9.3(-12.5) \times(1.0-$ )1.0-1.6 $(-2.0) \mu \mathrm{m}(n=20)$. Conidia abundant, single, hyaline to light brown, eguttulate, aseptate, allantoid, relatively large $(5.5-) 5.9-6.5(-7.0) \times(1.5-) 1.5-1.7(-2.0) \mu \mathrm{m}(n=30)$. No sexual morph observed.
Culture characteristics: Colonies after $7 \mathrm{~d}$ at $25^{\circ} \mathrm{C}$ on PDA average $63.7 \mathrm{~mm}$, medium-growing, white to off-white with sparse aerial tufts, peripheral hyphae submerged, hyphae becoming buff with age. Hyphae hyaline, smooth, straight, branched, and septate.

Distribution: San Joaquin County (California, USA).

Host: Olea europaea.

Notes: Based on the phylogenetic inference obtained in this study, C. pruinosa (isolated from Olea europaea var. africana in South Africa) is the closest relative to C. oleicola. Conidia of C. oleicola (5.5-)5.9-6.5(-7.0) × (1.5-)1.5-1.7(-2.0) $\mu \mathrm{m}$ are, on average, larger in terms of both length and width than conidia of $C$. pruinosa (5-6 × $1.2 \mu \mathrm{m}$; Adams et al. 2006).

Cytospora parakantschavelli Norphanph. et al., Mycosphere 8: 1 (2017).

(Figs 4 and 13)

Type: Russia: on branches and twigs of Populus $\times$ sibirica 12 May 2015, T. Bulgakov (MFLUCC 15-2094 - holotype).

Description: Conidiomata in PDA pycnidial, mostly solitary, rarely aggregated, globose, without conceptacle, black-grey 

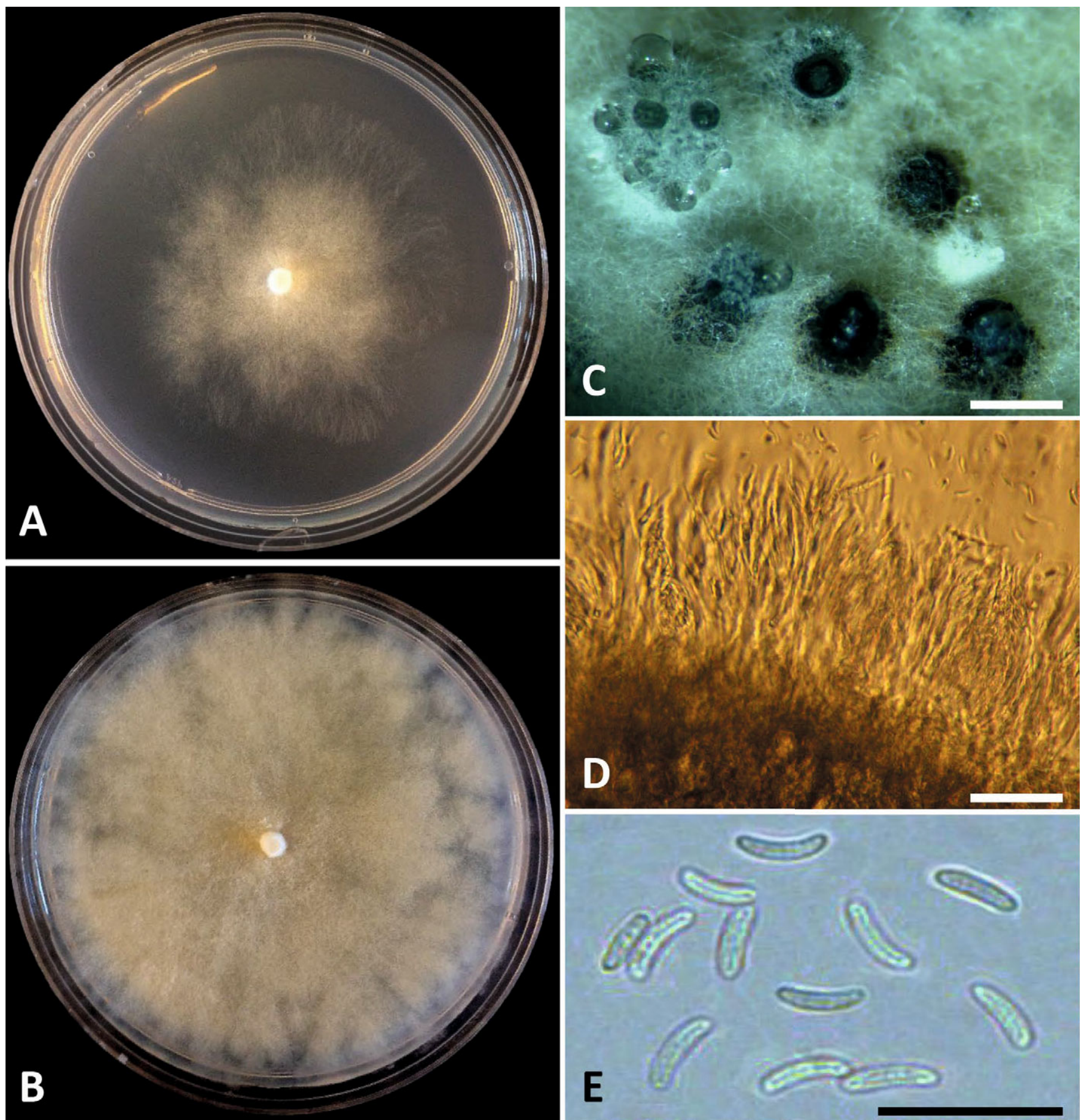

Fig. 12. Cytospora oleicola (ex-holotype culture CBS 144248). A. Seven-day-old PDA culture. B. Fourteen-day-old PDA culture. C. Pycnidia. D. Conidiophores and filamentous conidiogenous cells. E. Conidia. Bars $C=500 \mu \mathrm{m} ; \mathrm{D}=20 \mu \mathrm{m} ; \mathrm{E}=10 \mu \mathrm{m}$.

with off-white surface hyphae, (1215-)1381-2099(-2600) $\mu \mathrm{m}$ diam $(n=20)$, with a single internal locule. Conidiophores straight, slender, then branching into 3-4 conidiogenous cells $(6.0-) 6.9-9.5(-9.5) \times(1.0-) 1.1-1.5(-2.0) \mu \mathrm{m}(n=20)$. Conidia abundant, single, hyaline to light brown, eguttulate, aseptate, allantoid, (5.5-)6.0-7.0(-7.5) × (1.0-)1.2-1.6(-1.5) $\mu \mathrm{m}(n=30)$. No sexual morph observed.

Culture characteristics: Colony of $C$. parakantschavelli isolate KARE974 $70 \mathrm{~mm}$ diam in $7 \mathrm{~d}$ at $25^{\circ} \mathrm{C}$ on PDA, fast-growing, off-white with cream centre with short aerial tufts giving a cottony appearance, peripheral hyphae submerged, aerial hyphae becoming darker with age. Hyphae hyaline, smooth, straight, branched, and septate.

Distribution: Rostov Region, Russia and San Joaquin and Yolo Counties (California, USA).

Hosts: Populus deltoides, Populus freemontii, Populus ×sibirica, and Pyrus pyraster.

Notes: Based on the phylogenetic inference obtained in this study, C. salicicola and C. kantschavelli are the closest relatives to $C$. parakantschavellii. The name $C$. 

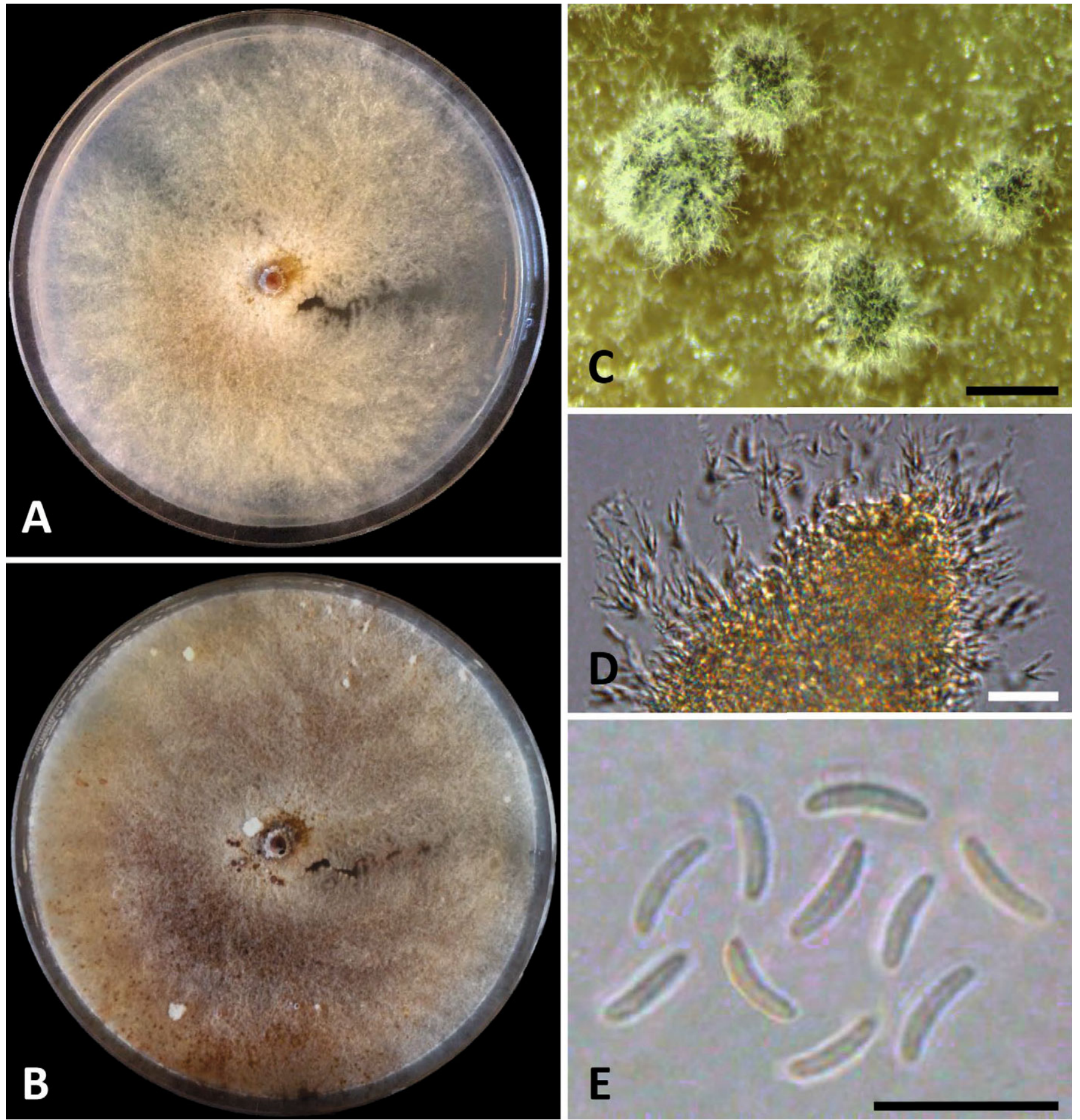

Fig. 13. Cytospora parakantschavelii (CBS 144243). A. Seven-day-old PDA culture. B. Fourteen-day-old PDA culture. C. Conidia. D. Conidiophores and filamentous conidiogenous cells. E. Pycnidia. Bars $C=20 \mu \mathrm{m} ; \mathrm{D}=10 \mu \mathrm{m} ; \mathrm{E}=1 \mathrm{~mm}$.

parakantschavellii was recently introduced by Norphanphoun et al. (2017) from Populus and Pyrus in Russia.

Additional specimen examined: USA, California: San Joaquin County, isolated from wood canker of Prunus dulcis, $21 \mathrm{Apr}$. 2016, F.P. Trouillas KARE974 (BPI 910658 [dried culture]; CBS 144243).

Cytospora parapistaciae D.P. Lawr., L.A. Holland \& Trouillas, sp. nov.

MycoBank MB824280

(Figs 4 and 14)
Etymology: The name refers to the phylogenetic position of this fungus in relation to the sister taxon C. pistaciae.

Diagnosis: Cytospora parapistaciae is readily distinguished from C. pistaciae based on pycnidial shape (mostly solitary submerged $v s$. globose aggregated) and conidiogenous cells (single straight cells vs. 3-4 branching cells).

Type: USA: California: Kern County, isolated from wood canker of Pistacia vera, 26 June 2015, M.T. Nouri KARE270 (BPI 910659 [dried culture] - holotype; CBS 144506 - exholotype culture). 

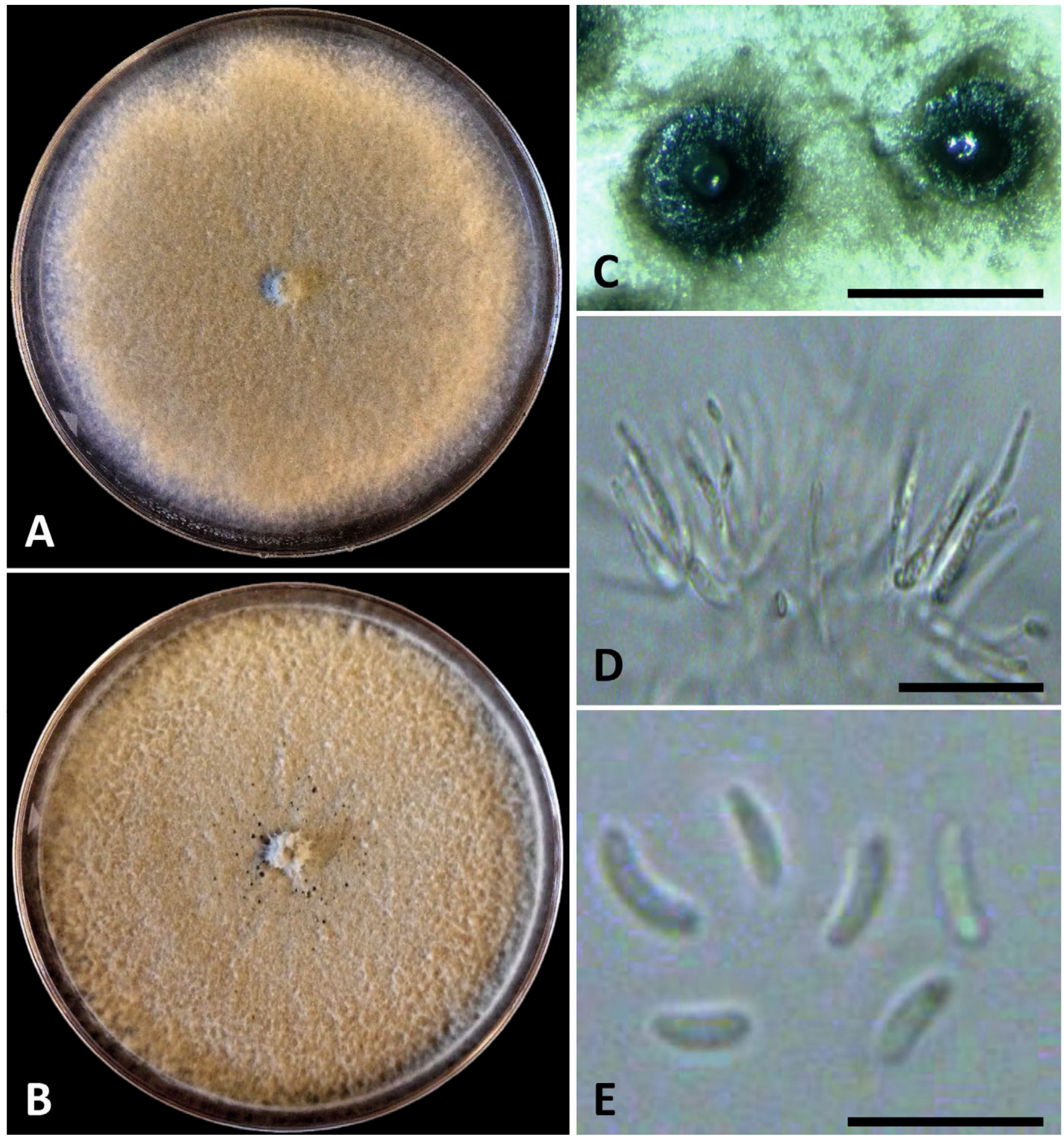

Fig. 14. Cytospora parapistaciae (ex-holotype culture CBS 144506). A. Seven-day-old PDA culture. B. Fourteen-day-old PDA culture. C. Pycnidia. D. Conidiophores and filamentous conidiogenous cells. E. Conidia. Bars $C=500 \mu \mathrm{m}$; D-E $=10 \mu \mathrm{m}$.

Description: Conidiomata on PDA pycnidial, mostly solitary, rarely aggregated, submerged to partially submerged, without conceptacle, black-grey, (335-)390-550(-590) $\mu \mathrm{m}$ diam ( $n$ $=20$ ), with a single internal locule. Conidiophores hyaline, reduced to straight, slender, filamentous conidiogenous cells $(7.0-) 7.6-9.6(-11.0) \times(1.0-) 1.2-1.6(-2.0) \mu \mathrm{m}(n=20)$. Conidia abundant, single, hyaline to light brown, eguttulate, aseptate, allantoid, small, (3.0-)3.5-4.3(-4.5) × (1.0-)0.91.1(-1.5) $\mu \mathrm{m}(n=30)$. No sexual morph observed.
Culture characteristics: Colonies after $7 \mathrm{~d}$ at $25^{\circ} \mathrm{C}$ on PDA average $87.3 \mathrm{~mm}$, fast-growing, buff to honey with short aerial tufts giving a cottony appearance, aerial hyphae very dense becoming darker buff to honey with white margin with age. Hyphae hyaline to light brown, smooth, straight, branched, and septate.

\section{Distribution: Kern County (California, USA).}

Host: Pistacia vera. 
Notes: Based on the phylogenetic inference obtained in this study, C. pistaciae is the closest relative of C. parapistaciae, both of which originated from pistachio cankers in two separate counties in California.

Cytospora pistaciae D.P. Lawr., L.A. Holland \& Trouillas, sp. nov. MycoBank MB824281

(Figs 4 and 15)
Etymology: The name refers to the host, Pistacia vera, from which this fungus was first isolated.

Diagnosis: Cytospora pistaciae is readily distinguished from $C$. parapistaciae based on pycnidial shape (globose aggregated vs. mostly solitary submerged) and conidiogenous cells (3-4 branching cells vs. single straight cells).

Type: USA: California: Merced County, isolated from wood canker of Pistacia vera, 14 Oct. 2015, F.P. Trouillas KARE443
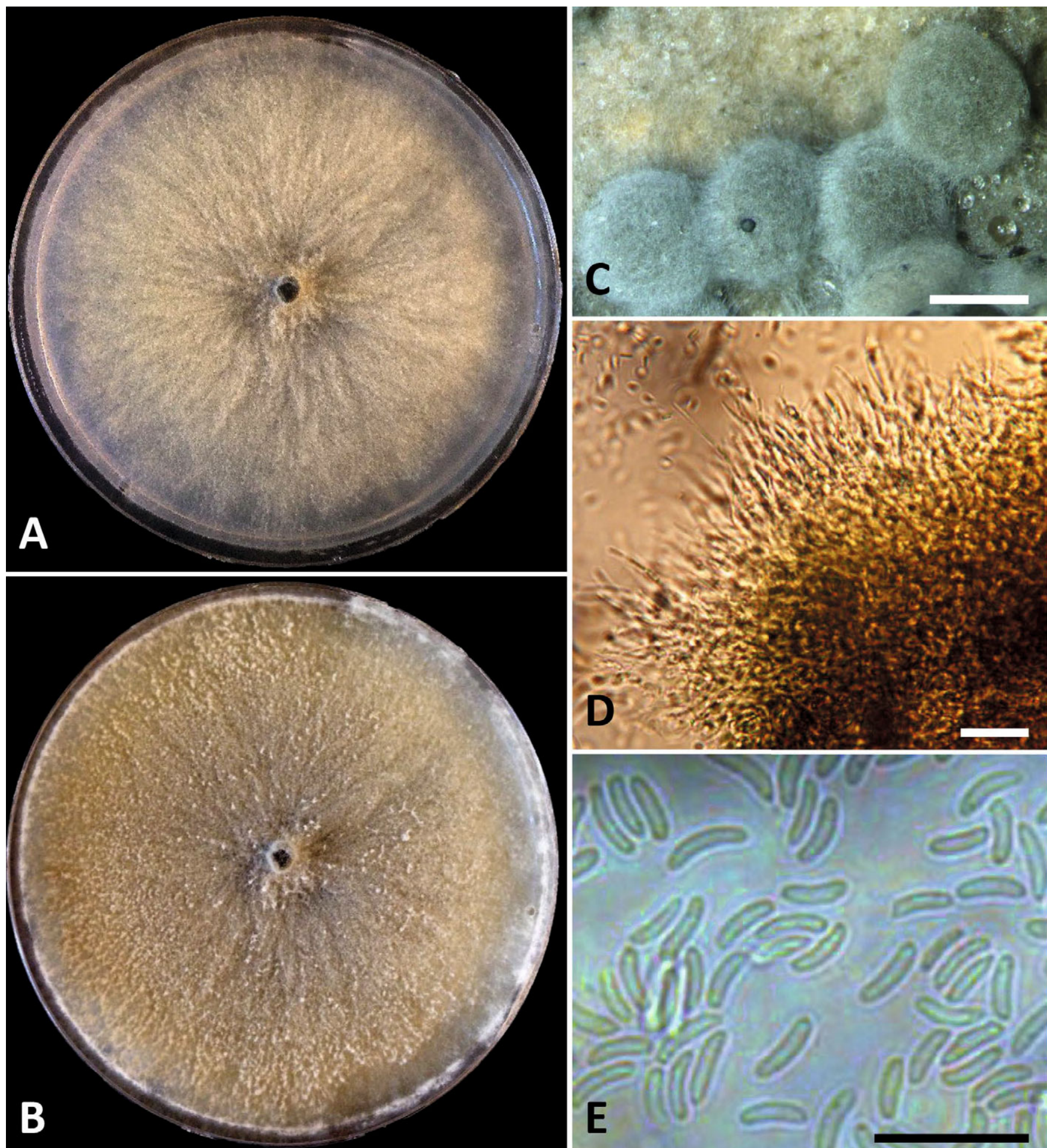

Fig. 15. Cytospora pistaciae (ex-holotype culture CBS 144238). A. Seven-day-old PDA culture. B. Fourteen-day-old PDA culture. C. Pycnidia. D. Conidiophores and filamentous conidiogenous cells. E. Conidia. Bars $C=1 \mathrm{~mm} ; \mathrm{D}=10 \mu \mathrm{m} ; \mathrm{E}=20 \mu \mathrm{m}$. 
(BPI 910660 [dried culture] - holotype; CBS 144238 - exholotype culture).

Description: Conidiomata on PDA pycnidial, solitary to regularly aggregated, globose, without conceptacle, light mouse-grey, (975-)1196-2184(-2655) $\mu \mathrm{m}$ diam $(n=20)$, with a single internal locule. Conidiophores straight, reduced to 3-4 branching filamentous conidiogenous cells (5.5-) 7.1-8.9(-10.0) × (1.0-)1.1-1.5(-2.0) $\mu \mathrm{m}(n=20)$. Conidia abundant, single, hyaline, eguttulate, aseptate, allantoid, $(3.5-) 4.0-4.8(-5.5) \times(1.0-) 1.1-1.3(-1.5) \mu \mathrm{m}(n=30)$. No sexual morph observed.

Culture characteristics: Colonies after $7 \mathrm{~d}$ at $25^{\circ} \mathrm{C}$ on PDA average $87.3 \mathrm{~mm}$, fast-growing, buff becoming honey with short aerial tufts giving a cottony appearance, peripheral hyphae submerged, aerial hyphae becoming darker with age. Hyphae hyaline, smooth, straight, branched, and septate.

Distribution: Merced County (California, USA).
Notes: Based on the phylogenetic inference obtained in this study, C. parapistaciae is the closest relative of $C$. pistaciae.

Cytospora plurivora D.P. Lawr., L.A. Holland \& Trouillas, sp. nov.

MycoBank MB824282

(Figs 4 and 16)

Etymology: The name refers to the plethora of hosts this fungus was routinely isolated from.

Diagnosis: Cytospora plurivora is distinguished from $C$. amygdali and $C$. erumpens in the smaller conidia in terms of length and width.

Type: USA: California: San Joaquin County, isolated from twig lesions of Olea europaea, 24 June 2016, F.P. Trouillas KARE1452 (BPI 910661 [dried culture] - holotype; CBS 144239 - ex-holotype culture).

Description: Conidiomata on PDA pycnidial, large, some solitary, many gregarious, globose to extended globose,
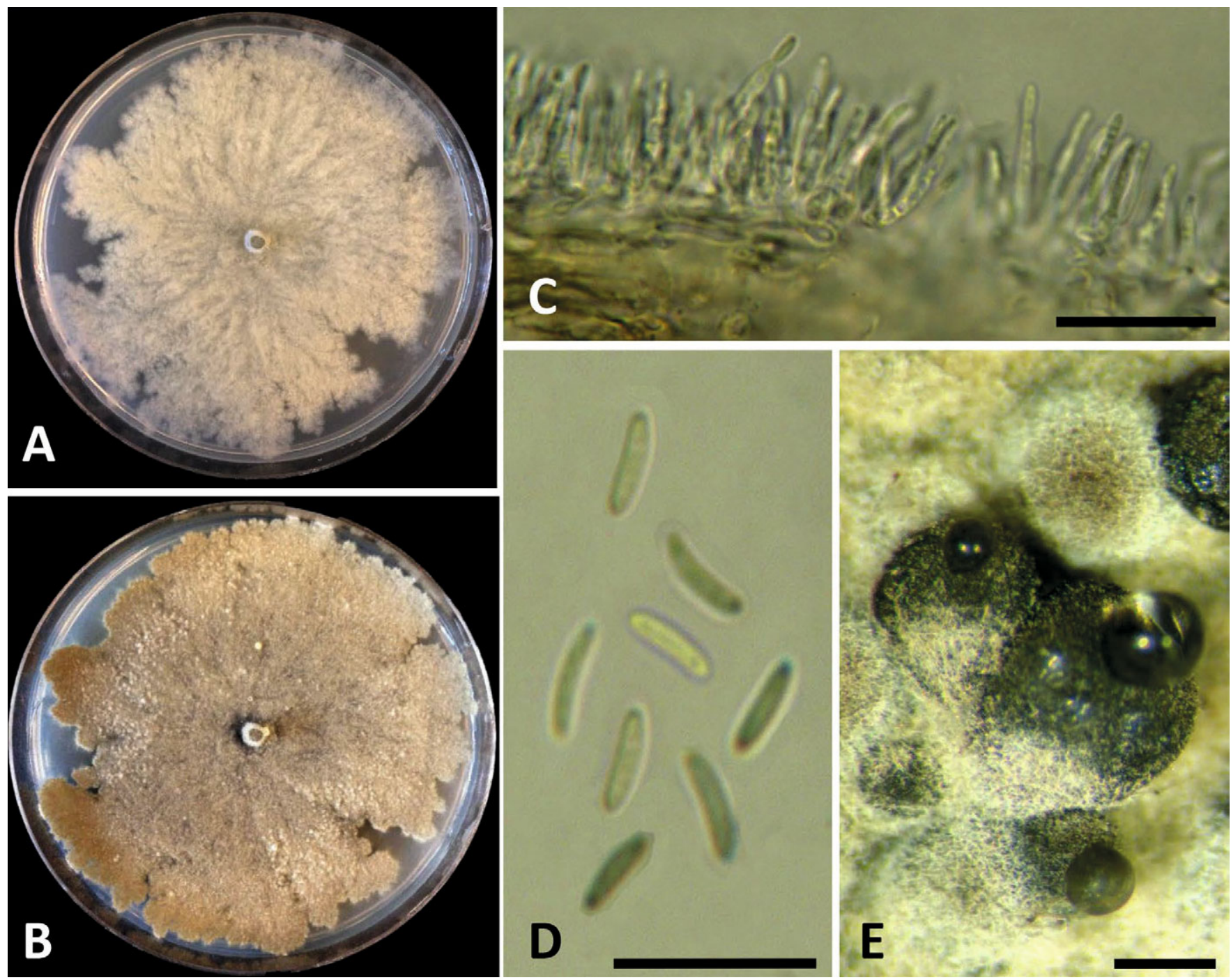

Fig. 16. Cytospora plurivora (ex-holotype culture CBS 144239). A. Seven-day-old PDA culture. B. Fourteen-day-old PDA culture. C. Conidiophores and filamentous conidiogenous cells. D. Conidia. E. Pycnidia. Bars $C=20 \mu \mathrm{m} ; \mathrm{D}=10 \mu \mathrm{m} ; \mathrm{E}=1 \mathrm{~mm}$. 
no conceptacle, black-grey with off-white surface hyphae, (1110-)1152-1968(-2745) $\mu \mathrm{m}$ diam $(n=20)$, with a single internal locule. Conidiophores reduced to single, straight, slender, filamentous conidiogenous cells (7.0-)7.7-10.0($11.0) \times(1.0-) 1.0-1.4(-1.5) \mu \mathrm{m}(n=20)$. Conidia abundant, single, hyaline to dark brown, eguttulate, aseptate, allantoid, $(3.5-) 3.8-4.4(-4.5) \times(1.0-) 0.9-1.1(-1.5) \mu \mathrm{m}(n=30)$. No sexual morph observed.

Culture characteristics: Colonies after $7 \mathrm{~d}$ at $25^{\circ} \mathrm{C}$ on PDA average $82 \mathrm{~mm}$, fast-growing, uneven lobate growth margin, off-white to cream with short aerial tufts giving a cottony appearance, aerial hyphae becoming light brown with age. Hyphae hyaline, smooth, straight, branched, and septate.

Distribution: Butte, Colusa, Contra Costa, Fresno, Glenn, Kern, San Joaquin, Stanislaus, Sutter, Tehama, Tulare, and Yuba Counties (California, USA).

Hosts: Juglans regia, Olea europaea, Pistacia vera, Prunus domestica, Prunus dulcis, and Prunus persica.

Notes: Based on the phylogenetic inference obtained in this study, C. amygdali is the closest species to C. plurivora, albeit with no statistical support. Cytospora plurivora is the most genetically diverse clade identified in this study which in part is likely due to its incidence on many different fruit and nut crop hosts throughout California.

Cytospora populicola D.P. Lawr., L.A. Holland \& Trouillas, sp. nov.

MycoBank MB824283

(Figs 4 and 17)

Etymology: The name refers to the host Populus and -cola for inhabitor.

Diagnosis: Cytospora populicola is distinguished from $C$. longiostiolata and $C$. rostrata in the shorter conidia than $C$. longiostiolata and larger conidia than $C$. rostrata, respectively.

Type: USA: California: San Joaquin County, isolated from wood canker of Populus deltoides, 21 Apr. 2016, F.P. Trouillas KARE973 (BPI 910662 [dried culture] - holotype; CBS 144240 - ex-holotype culture).

Description: Conidiomata on PDA pycnidial, mostly solitary, rarely aggregated, some with yellow conidial exudate, globose to conical, without conceptacle, black-grey, (1015-) 1210-2210 $(-2735) \mu \mathrm{m}$ diam $(n=20)$, with a single internal locule. Conidiophores reduced to 3-4 filamentous branching conidiogenous cells tapering toward apices (5.5-)6.1-8.1(-10.0) $\times(1.0-) 1.5-1.9(-2.0) \mu \mathrm{m}(n=20)$. Conidia abundant, single, hyaline, eguttulate, aseptate, allantoid, (4.5-)4.7-5.3(-5.5) $\times$ (1.0-)1.1-1.4(-1.5) $\mu \mathrm{m}(n=30)$. No sexual morph observed.

Culture characteristics: Colonies after $7 \mathrm{~d}$ at $25^{\circ} \mathrm{C}$ on PDA average $87.3 \mathrm{~mm}$, medium-growing with uneven margin expansion, off-white with short aerial tufts giving a cottony appearance, aerial hyphae becoming cream-coloured with age. Hyphae hyaline, smooth, straight, branched, and septate. Distribution: San Joaquin County (California, USA).

Host: Populus deltoides.

Notes: Based on the phylogenetic inference obtained in this study, C. longiostiolata and C. rostrata, both isolated from Salix, are the closest species to $C$. populicola. Conidia of $C$. populicola are, on average, larger than those of $C$. rostrata 3.6-4.8 $\times 1.0-1.6 \mu \mathrm{m}$ (av. $4.1 \times 1.4 \mu \mathrm{m}$ ) and smaller than those of C. longiostiolata (3.9)5.4-6.6 × 1.0-1.2(-1.5) (av. $5.5 \times 1.3 \mu \mathrm{m})$.

Cytospora punicae Sacc., Michelia 1: 367 (1878) ; as 'punica'.

Figs 4 and 18.

Description: Conidiomata on PDA pycnidial, gregarious, globose to subglobose, no conceptacle, black-grey with offwhite surface hyphae, (210-)237-383(-490) $\mu \mathrm{m}$ diam $(n=20)$, with multiple internal locules with shared invaginated walls. Conidiophores single, straight, filamentous conidiogenous cells (5.5-)5.8-8.6(-9.5) x (1.0-)1.1-1.4(-2.0) $\mu \mathrm{m}(n=20)$. Conidia abundant, single, hyaline to light brown, eguttulate, aseptate, allantoid, (3.5-)3.8-4.6(-5.0) × (0.5-)0.8-1.0(-1.0) $\mu \mathrm{m}(n=30)$. No sexual morph observed.

Culture characteristics: Colony of C. punicae isolate 5A-80 $64.7 \mathrm{~mm}$ diam in $7 \mathrm{~d}$ at $25{ }^{\circ} \mathrm{C}$ on PDA. Medium-growing, dark red becoming lighter with age. Hyphae hyaline, smooth, straight, branched, and septate.

Distribution: Fresno, Madera, and Stanislaus Counties (California, USA), Cyprus, Greece, Iran, South Africa, and Turkey.

Host: Punica granatum.

Notes: Based on the phylogenetic inference obtained in this study, C. myrtagena is the closest species to $C$. punicae. Only two species of Cytospora are known from pomegranate (C. granati and C. punicae) and these can be distinguished by the diagnostic red hyphae/colony of $C$. punicae in culture. The colony growth of Cytospora punicae is also much slower (64.7 $\mathrm{mm}$ in $7 \mathrm{~d}$ ) compared to $C$. granati $(87.3 \mathrm{~mm}$ in $7 \mathrm{~d}$ ).

Specimen examined: USA: California: Madera County, isolated from wood canker of Punica granatum, 21 July 2010, T.J. Michailides 5A80 (BPI 910663 [dried culture]; CBS 144244).

Cytospora sorbicola Norphanph. et al., Mycosphere 8: 1 (2017).

Figs 4 and 19 .

Type: Russia: on dead and dying branches of Acer pseudoplatanus 18 June 2015, T. Bulgakov (MFLUCC 152203 - holotype).

Description: Conidiomata on PDA pycnidial, mostly solitary, sometimes aggregated, globose, without conceptacle, 

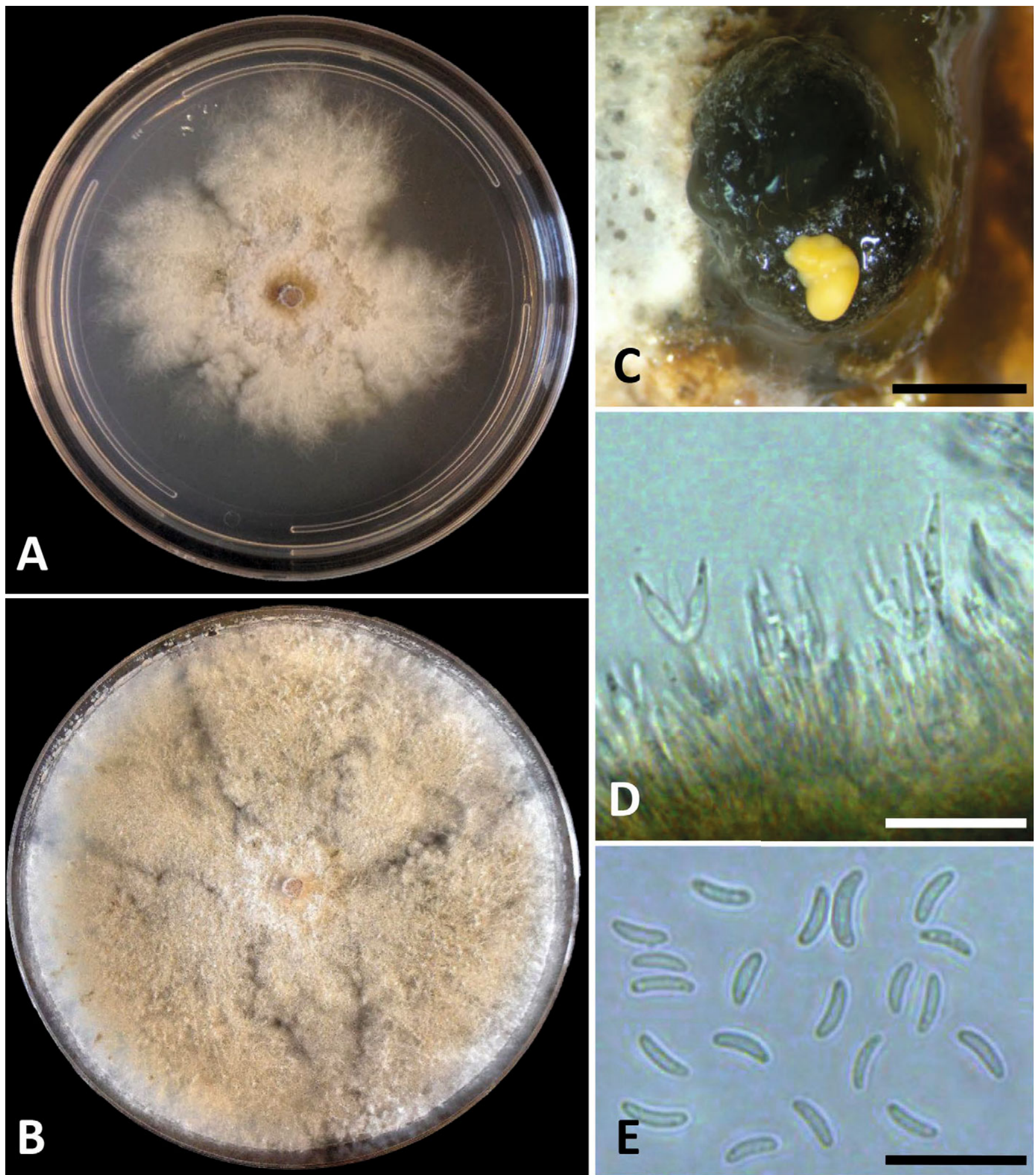

Fig. 17. Cytospora populicola (ex-holotype culture CBS 144240). A. Seven-day-old PDA culture. B. Fourteen-day-old PDA culture. C. Pycnidia. D. Conidiophores and filamentous conidiogenous cells. E. Conidia. Bars $C=500 \mu \mathrm{m}$; D-E $=10 \mu \mathrm{m}$.

mouse-grey, (1020-)1220-1900(-2420) $\mu \mathrm{m}$ diam $(n=20)$ with 1-2 locules. Conidiophores branched, reduced to filamentous conidiogenous cells that taper towards the apices $(4.5-) 6.4-9.6(-10.0) \times(1.0-) 1.0-1.4(-2.0) \mu \mathrm{m}(n=20)$. Conidia abundant, single, hyaline to light brown, eguttulate, aseptate, allantoid, (3.5-)4.0-4.6(-4.5) × (1.0-)0.9-1.1(-1.0) $\mu \mathrm{m}(n=30)$. No sexual morph observed.
Culture characteristics: Colony of $C$. sorbicola isolate KARE228 $81.7 \mathrm{~mm}$ diam in $7 \mathrm{~d}$ at $25{ }^{\circ} \mathrm{C}$ on PDA, fastgrowing, off-white to cream with general lack of aerial hyphae, colony darkens with age. Hyphae hyaline, smooth, straight, branched, and septate.

Distribution: Contra Costa, Fresno, Kings, Merced, Sacramento, San Benito, San Joaquin, Stanislaus, Yolo, and 

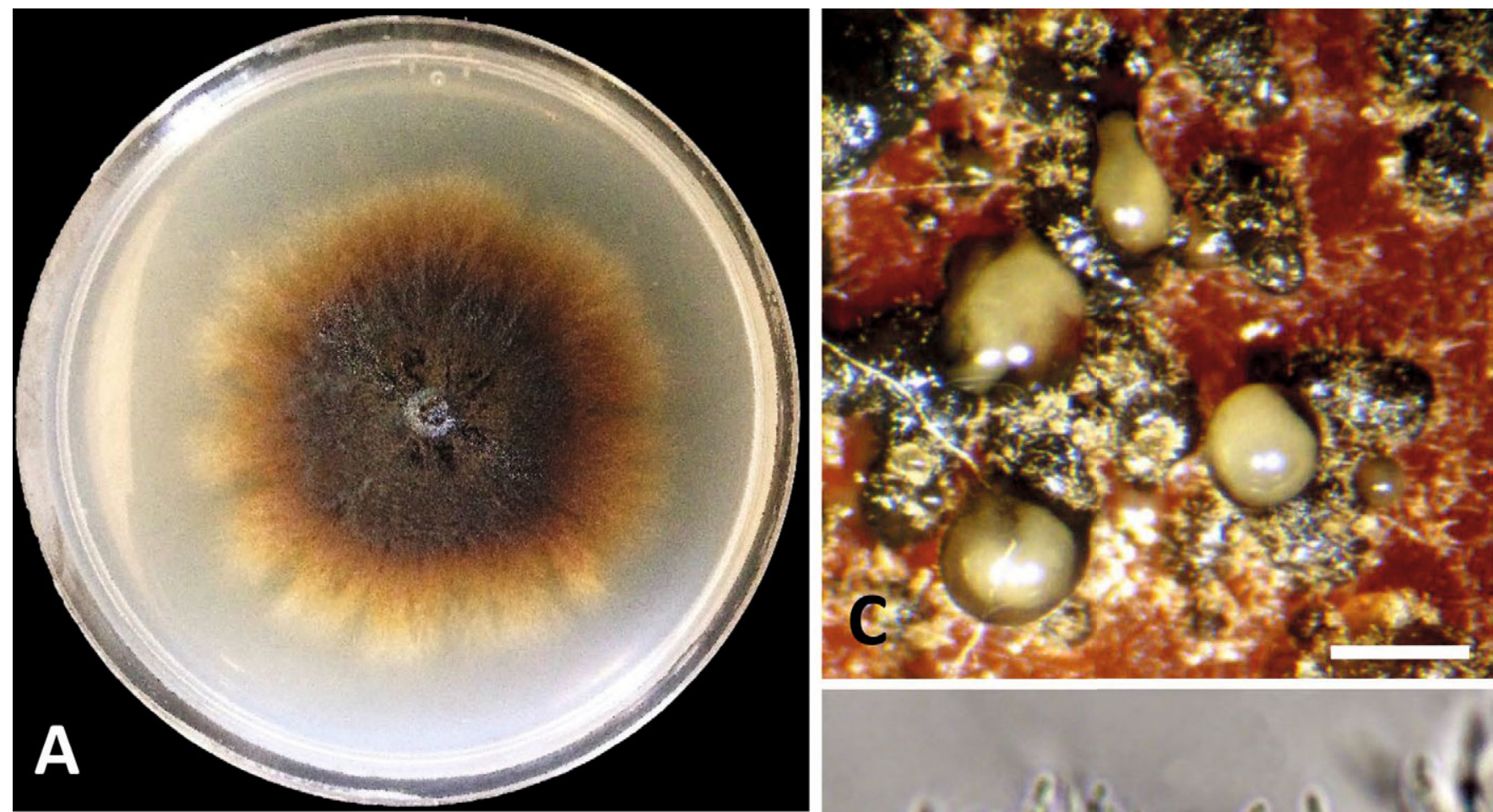

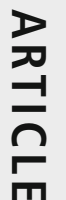
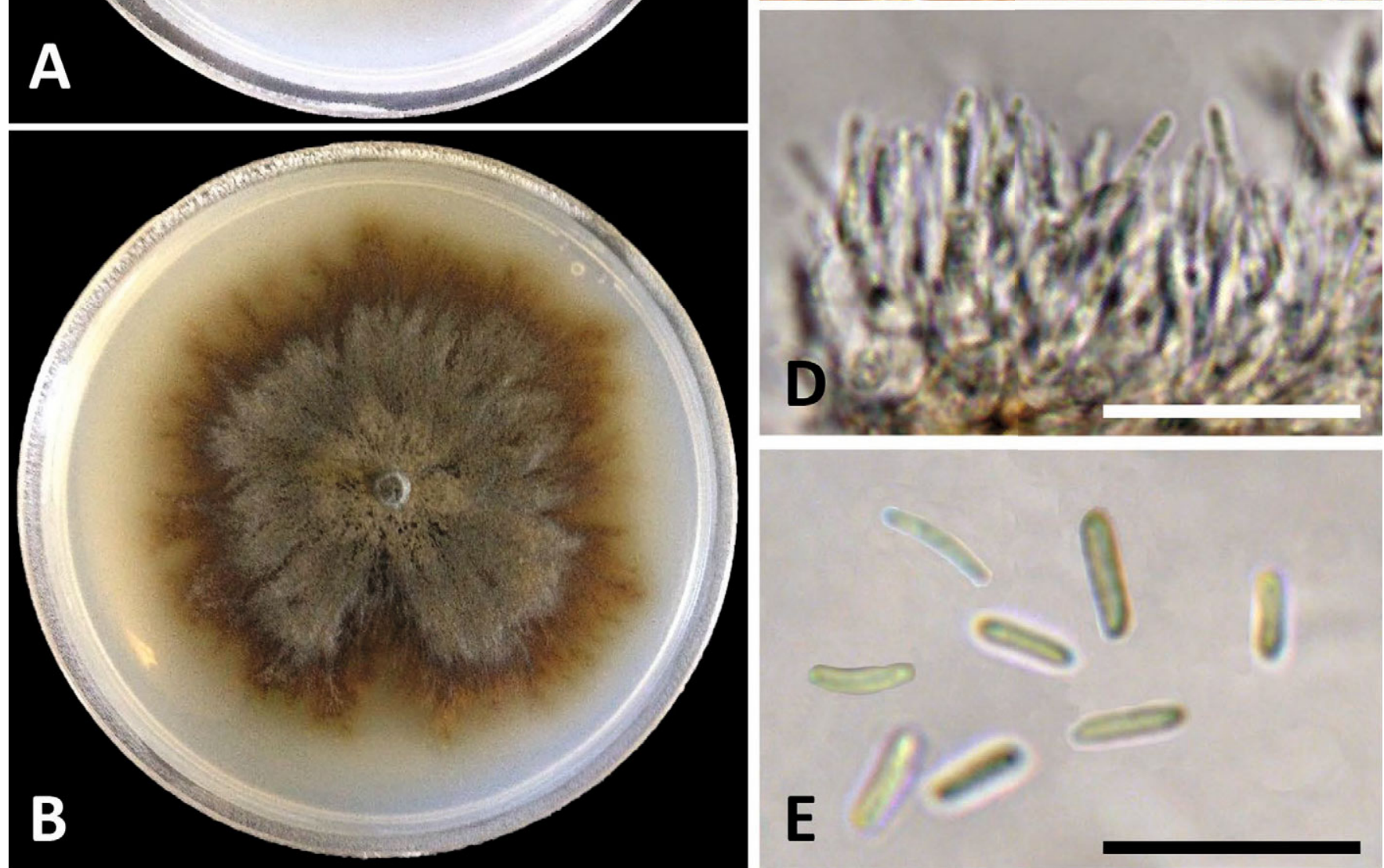

Fig. 18. Cytospora punicae (CBS 144244). A. Seven-day-old PDA culture. B. Fourteen-day-old PDA culture. C. Pycnidia. D. Conidiophores and filamentous conidiogenous cells. E. Conidia. Bars $C=500 \mu \mathrm{m} ; \mathrm{D}=20 \mu \mathrm{m} ; E=10 \mu \mathrm{m}$.

Yuba Counties (California, USA), and Rostov Region, Russia.

Hosts: Acer pseudoplatanus, Cotonoeaster melanocarpus, Prunus armeniaca, $P$. avium, $P$. cerasus, $P$. domestica, $P$. dulcis, P. persica, and Sorbaronia mitschurinii.

Notes: Based on the phylogenetic inference obtained in this study, $C$. donetzica is the closest species to $C$. sorbicola. The C. sorbicola isolates collected in this study display some host affiliation with cherry, clustering strongly in the MP analysis and no support in the ML analysis. The level of support for the California-only $C$. sorbicola isolates and differences in morphology suggests that they may represent a distinct lineage sister to $C$. sorbicola collected in Russia. Additional data such as TEF1 and TUB2 from the Russian type of $C$. sorbicola will help answer this question.

Additional specimen examined: USA, California: Stanislaus County, isolated from bark canker of Prunus dulcis, 15 July 2015, M.T. Nouri KARE228 (BPI 910664 [dried culture]; CBS 144245). 

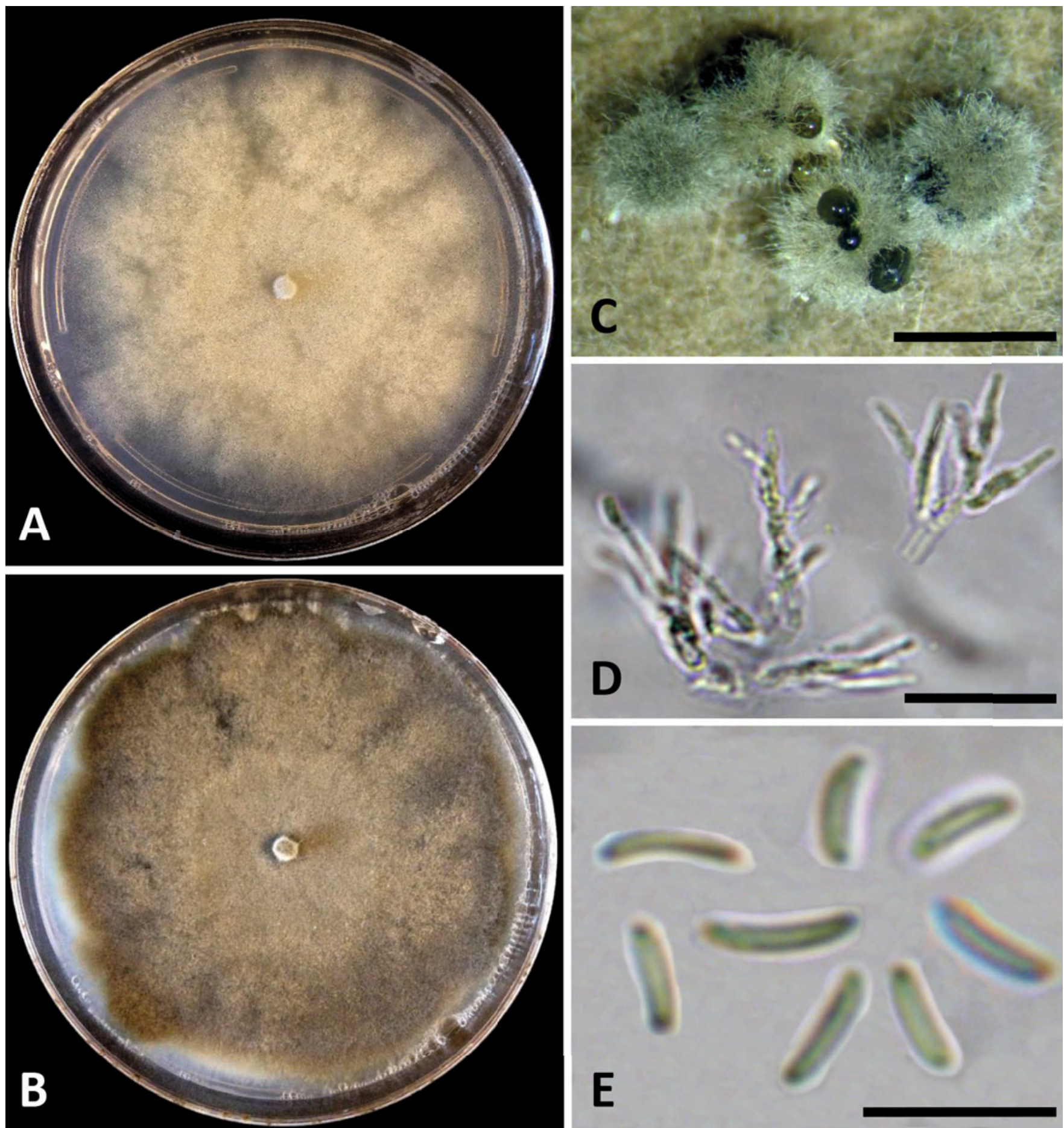

Fig. 19. Cytospora sorbicola (CBS 144245). A. Seven-day-old PDA culture. B. Fourteen-day-old PDA culture. C. Pycnidia. D. Conidiophores and filamentous conidiogenous cells. E. Conidia. Bars $C=1 \mathrm{~mm} ; \mathrm{D}=20 \mu \mathrm{m} ; \mathrm{E}=5 \mu \mathrm{m}$.

\section{DISCUSSION}

This manuscript presents a comprehensive molecular phylogenetic overview of Cytospora species currently known from culture, which was initiated due to a high incidence of Cytospora species associated with canker symptoms across diverse orchard crops in California. All Cytospora species known from culture and linked to publicly-available molecular data were considered for phylogenetic analyses in this study. The lack of ex-type cultures or sequence data for many species names makes it difficult to assess many older species names, especially for those described only by morphology. We deposited ten new ex-type specimens with two different public fungal biodiversity repositories, the Mycology and Nematology Genetic Diversity and Biology Laboratory in Beltsville, MD (BPI), and the Westerdijk Fungal Biodiversity Institute in The Netherlands (CBS), in conjunction with molecular data in GenBank, in order to strengthen and stabilize the taxonomy of Cytospora and to aid in the identification of Cytospora species via DNA sequence data in future studies by other mycologists and plant pathologists. 
Cytospora species are ubiquitous, important pathogens of many woody hosts causing cankers, dieback and mortality of forest and urban trees (Adams et al. 2005, 2006, Worrall et al. 2010) and of many economically important crops including Juglans, Malus, Prunus, and Vitis (Biggs \& Grove 2005, Wang et al. 2011, Fan et al. 2015a, Lawrence et al. 2017a). Results from this study unveiled 15 species of Cytospora from infected orchard crops and adjacent ornamentals in the Central Valley of California. These species include the previously described taxa C. chrysosperma, C. parakantschavelli, C. punicae, and $C$. sorbicola and 10 previously undescribed taxa names which are newly introduced: C. amygdali, C. californica, C. granati, C. joaquinensis, C. longispora, C. oleicola, C. parapistaciae, C. pistaciae, C. plurivora, and C. populicola, and a new combination, $C$. eucalypti. All species were strongly supported by both DNA sequence data and morphological observations. This study reports C. parakantschavelii and C. sorbicola for the first time in North America, including new host records for each species, Populus deltoides and $P$. freemontii for $C$. parakantschavelii and Olea europaea, Prunus avium, $P$. domestica, $P$. dulcis, and $P$. persica for $C$. sorbicola. Our Californian Cytospora eucalypti (syn. Valsa eucalypti) isolates cluster strongly with an isolate from the coastal redwoods (Sequoia sempervirens) reported in Adams et al. (2005), which also clusters strongly with isolates previously referred to as Valsa eucalypti, isolated from four species of Eucalyptus in California (Adams et al. 2006). This study expanded the known host range of $C$. eucalypti to include Prunus dulcis and Sequoiadendron gigateum (giant sequoia) in California.

The utility of asexual morph characters for species recognition has been questioned in Cytospora. Locule morphology seems to be influenced by the depth in the bark at which the pycnidia form, with variations from unilocular cytosporoid when formed deep in the bark to rosette cytosporoid when formed near the bark surface (Adams et al. 2005). Also, asexual morphs that form in nature can vary considerably from those forming in culture, and these morphological characters are not necessarily taxonomically informative (Adams et al. 2005). Considering that sexual morphs are rarely found in nature, the use of sexual morph morphology in species diagnosis has been limited. Furthermore, both ascospores and conidia of many Cytospora species are of similar shapes (single, allantoid, and aseptate) and sizes (4-8 × 1-2 $\mu \mathrm{m})$ thus complicating morphological separation of distinct lineages (Adams et al. 2002, 2005, Wang et al. 2011). In this study, we found the morphological characteristics of the conidia were indistinguishable among most species, with similar dimensions among the examined species; most asexual morph characters were not taxonomically informative.

The genus Cytospora includes both generalist pathogens (i.e. C. chrysosperma with 265 host records; USDA Fungus-Host Distribution Database, https://nt.ars-grin.gov/ fungaldatabases/fungushost/fungushost.cfm) and specialist pathogens (i.e. C. punicae with only one host record in the same USDA Database). As such, host associations do not appear to constitute an appropriate criterion for species recognition, as previously discussed (Adams et al. 2005, 2006). In this study, host association was not found to be taxonomically informative as many Cytospora species were recovered from multiple hosts. However, our work highlighted a few instances of close host associations. Prior to this study, C. punicae had been reported causing wood canker on pomegranate trees in California, Cyprus, and Iran (Peduto Hand et al. 2014, Samouel \& Kanetis 2016, Mahdikhani \& Davoodi 2017), pomegranate collar rot in Greece (Palavouzis et al. 2015), and pomegranate fruit rot in South Africa (Venter et al. 2017). Cytospora punicae was only recovered from pomegranate trees in this study, supporting this species as host specific despite a wide geographical distribution. Pomegranate trees harboured a second species, C. granati, which was only recovered from this host. Both $C$. punicae and C. granati have similar conidial shapes and dimensions, but the species have distinct pycnidial shapes and sizes and colony morphologies. Thus, host association paired with morphological observations may have utility when examining Cytospora species on pomegranate. In contrast, C. sorbicola was isolated from six hosts (almond, apricot, cherry, olive, peach, and plum) and these hosts typically harboured more than one Cytospora species. Within the C. sorbicola clade, a subclade strongly supported by parsimony analysis ( $86 \%$ ) but showing low support by likelihood analysis (<70 \%) contained isolates that originated almost exclusively from cherry. These findings suggest some level of genetic divergence for $C$. sorbicola isolates from cherry, which could indicate some host specialization in these isolates; a preliminary step towards reproductive isolation and ecological speciation (Giraud et al. 2010).

Given the variability, plasticity, and complexity of morphological characters in the genus (e.g. stromatal arrangement in the host tissues, locular arrangement within pycnidia, locule division into chambers, independent or shared locular walls), previous studies have advocated the use of molecular data to accurately identify Cytospora species (Adams et al. 2002, 2005, 2006). In this study, we used molecular phylogenetic analyses of four loci (ITS+TUB2+TEF1+ACT1), not only to identify species but also to provide reference data for future phylogenetic studies. Before this study, most Cytospora sequences deposited in GenBank consisted of ITS. While ITS is the primary marker for fungal barcoding (Schoch et al. 2012), in some fungal groups, ITS has insufficient power for species recognition whereas protein-coding genes can be more informative sequence regions for species delineation (O'Donnell et al. 2015, Lawrence et al. 2017b). For instance, analyses of TEF1 sequence data provided more discriminatory power than ITS in delineating two recently described Cytospora species occurring on grapevine, C. vinacea and C. viticola (Lawrence et al. 2017a). In other xylophilous fungi, 'secondary barcodes' such as TUB2, TEF1, and histone 3 (HIS) can also be preferable based on their ability to delineate closely related or cryptic species and on the availability of sequence data for ex-type specimens. For example TUB2 is the preferred marker for identification of fungi in the Togniniaceae (i.e. Phaeoacremonium minimum) and TEF1 is the preferred marker for the Botryosphaeriaceae (i.e. Neofusicoccum parvum) and Diaporthales (which includes Cytosporaceae) (Lawrence et al. 2017b), especially for closely related or cryptic species. In agreement with previous studies (Adams 
et al. 2002, 2005), our findings revealed that ITS has sufficient power to discriminate the 15 Cytospora species reported from orchard crops in California. However, based on comparisons of clade support values of each locus used in this study, it appears that TEF1 is the preferential locus to use for Cytospora identification as it was able to strongly support all 15 lineages in this study. Moreover, in our study, 362/799 (45 $\%$ ) of the aligned nucleotide positions in TEF1 and 142/365 (39 \%) in ACT1 were parsimony informative, whereas only 119/575 (21\%) and 180/604 (30\%) were parsimony informative in TUB2 and ITS, respectively. Therefore, a DNAbased approach utilizing several gene regions (in order of priority: TEF1, ACT1, ITS, and TUB2 using the primer pairs in this study) would be the best method to resolve Cytospora species concepts, especially when morphological characters and host occurrences may be misleading due to significant overlap.

Until the present study, the diversity of Cytospora species affecting perennial crops in California has been largely overlooked and underestimated. Historically, two species, C. cincta and C. leucostoma, have been associated with Cytospora canker of stone fruits and pome fruits in North America (Bertrand \& English 1976b, Biggs 1989, Biggs \& Grove 2005). Surprisingly, we did not isolate either species in this study, suggesting that $C$. cincta and $C$. leucostoma were originally misidentified as the causal agents of Cytospora canker of stone fruits and pome fruits in California. Our findings suggest that many species of Cytospora are involved in the decline of fruit and nut crops in California, and they do not include either $C$. cincta nor $C$. leucostoma. The main putative causal agents of Cytospora canker of stone fruits (apricot, cherry, peach, and prune) in California included $C$. plurivora and C. sorbicola. Similarly, the main putative causal agents of Cytospora canker of nut crops (almond, pistachio, and walnut) in California included C. amygdali, C. californica, C. eucalypti, C. joaquinensis, C. parapistaciae, C. pistaciae, C. plurivora, and C. sorbicola. Three species were associated with Cytospora canker of Populus trees, C. joaquinensis, C. parakantschvelii, and C. populicola. Cytospora joaquinensis was also associated with cankers in pistachio and walnut, suggesting that cross infections occur between orchards and adjacent ornamentals and vice versa. Three species were associated with Cytospora canker of olive (C. oleicola, C. plurivora, and $C$. sorbicola) with the two latter species also collected from other hosts. Two species were exclusively associated with Cytospora canker of pomegranate ( $C$. granati and $C$. punicae). These results strongly suggest the need for additional research concerning the epidemiology of Cytospora species that cause Cytospora canker in fruit and nut crops and proximal ornamentals in the diverse agricultural areas of the Central Valley of California.

Research on Cytospora canker of stone fruits had received broad attention before the advent of molecular identification of fungi, focusing on seasonal activities of pathogenic species (Bertrand \& English 1976a), spore production (Bertrand \& English 1976b), etiology, epidemiology and host resistance (Biggs 1989). According to our findings, pathogenicity studies should now be conducted to elucidate the role of the newly described Cytospora species in the fruit and nut crops in California. The large diversity of species revealed in this study also suggests that management of Cytospora canker needs to be re-evaluated following accurate molecular identification to determine the main pathogenic species involved within each crop. Control of Cytospora diseases is difficult and focusing management efforts against the most aggressive encountered Cytospora species will be essential. The genus Cytospora represents a good example of a fungal group where morphological features are extremely complex and not necessarily informative from a taxonomic standpoint, which could in part explain why in North America only two species were previously considered the main causal agents of Cytospora canker of perennial crops. This study constitutes a further step towards a sequencebased description of fungal species in an important group of plant pathogens, revealing a large species richness, providing type specimens associated with molecular data for new taxa, detailed morphological descriptions, and some evidence for appropriate selection of loci for molecular typing. Furthermore, this study provides a firm foundation for future pathogenicity, ecological, and epidemiological studies to better help manage canker diseases in perennial crops infected by Cytospora species.

\section{ACKNOWLEGEMENTS}

This manuscript is dedicated to the 200-year-old generic name Cytospora. We thank the California Cherry Board, the California Pistachio Research Board and the Almond Board of California for financial support. We thank also Francesca Peduto-Hand for supplying images of Cytospora canker of pomegranate.

\section{REFERENCES}

Adams GC, Roux J, Wingfield MJ (2006) Cytospora species (Ascomycota, Diaporthales, Valsaceae): introduced and native pathogens of trees in South Africa. Australasian Plant Pathology 35: 521-548.

Adams GC, Surve-lyer RS, lezzoni AF (2002) Ribosomal DNA sequence divergence and group I introns within the Leucostoma species L. cinctum, L. persoonii, and L. parapersoonii sp. nov., ascomycetes that cause Cytospora canker of fruit trees. Mycologia 94: 947-967.

Adams GC, Wingfield MJ, Common R, Roux J (2005) Phylogenetic relationships and morphology of Cytospora species and related teleomorphs (Ascomycota, Diaporthales, Valsaceae) from Eucalyptus. Studies in Mycology 52: 1-144.

Alves A, Crous PW, Correia A, Phillips A (2008) Morphological and molecular data reveal cryptic speciation in Lasiodiplodia theobromae. Fungal Diversity 28: 1-13.

Bertrand P, English H (1976a) Virulence and seasonal activity of Cytospora leucostoma and Cytospora cincta in French prune trees in California. Plant Disease Reporter 60: 106-110.

Bertrand PF, English H (1976b) Release and dispersal of conidia and ascospores of Valsa leucostoma. Phytopathology 66: 987-991.

Biggs AR (1989) Integrated approach to controlling Leucostoma canker of peach in Ontario. Plant Disease 73: 869-874.

Biggs AR, Grove GG (2005) Leucostoma canker of stone fruits. The Plant Health Instructor 10: DOI: 10.1094/PHI-I-2005-1220-1001. 
Bills GF (1996) Isolation and Analysis of Endophytic Fungal Communities from Woody Plants. St Paul, MN: American Phytopathological Society Press.

Carbone I, Kohn LM (1999) A method for designing primer sets for speciation studies in filamentous ascomycetes. Mycologia 91: 553-556.

Chang LS, lezzoni AF, Adams GC, Ewers FW (1991) Hydraulic conductance in susceptible versus tolerant peach seedlings infected with Leucostoma persoonii. Journal of the American Society for Horticultural Science 116: 831-834.

Christensen CM (1940) Studies on the biology of Valsa sordida and Cytospora chrysosperma. Phytopathology 30: 459-475.

Défago G (1935) De quelques Valsees von Höhnel parasites des arbres a noyau deperissants. Beitrage zur Kryptogamenflora von Schweiz 8: 1-111.

Fan X, Hyde KD, Liu M, Liang Y, Tian C (2015a) Cytospora species associated with walnut canker disease in China, with description of a new species C. gigalocus. Fungal Biology 119: 310-319.

Fan X, Hyde KD, Yang Q, Liang Y, Ma R, Tian C (2015b) Cytospora species associated with canker disease of three antidesertification plants in northwestern China. Phytotaxa 197: 227-244.

French AM (1989) California Plant Disease Host Index. Sacramento, CA: California Department of Food and Agriculture, Division of Plant Industry.

Giraud T, Gladieux P, Gavrilets S (2010) Linking the emergence of fungal plant diseases with ecological speciation. Trends in Ecology and Evolution 25: 387-395.

Giraud T, Villareal LM, Austerlitz F, Le Gac M, Lavigne C (2006) Importance of the life cycle in sympatric host race formation and speciation of pathogens. Phytopathology 96: 280-287.

Glass NL, Donaldson GC (1995) Development of primer sets designed for use with the PCR to amplify conserved genes from filamentous ascomycetes. Applied and Environmental Microbiology 61: 1323-1330.

Grove W (1923) The British species of Cytospora. Bulletin of Miscellaneous Information, Royal Botanic Gardens, Kew 1923: 1-30.

Hawksworth DL (2011) A new dawn for the naming of fungi: impacts of decisions made in Melbourne in July 2011 on the future publication and regulation of fungal names. IMA Fungus 2: 155162.

Hibbett D, Abarenkov K, Kõljalg U, Öpik M, Chai B, et al. (2016) Sequence-based classification and identification of Fungi. Mycologia 108: 1049-1068.

Kirk PM, Cannon PF, Minter DW, Stalpers JA (eds) (2008) Ainsworth \& Bisby's Dictionary of the Fungi. $10^{\text {th }}$ edn. Wallingford: $C A B$ International.

Lawrence DP, Travadon R, Baumgartner K (2015) Diversity of Diaporthe species associated with wood cankers of fruit and nut crops in northern California. Mycologia 107: 926-940.

Lawrence DP, Travadon R, Pouzoulet J, Rolshausen P, Wilcox W, Baumgartner K (2017a) Characterization of Cytospora isolates from wood cankers of declining grapevine in North America, with the descriptions of two new Cytospora species. Plant Pathology 66: 713-725.

Lawrence DP, Travadon R, Nita M, Baumgartner K (2017b) TrunkDiseaselD. org: A molecular database for fast and accurate identification of fungi commonly isolated from grapevine wood. Crop Protection 102: 110-117.
Maddison WP, Maddison DR (2016) Mesquite: a modular system for evolutionary analysis. Version 3.10. http://mesquiteproject.org.

Mahdikhani M, Davoodi A (2017) First report of wood canker of pomegranate caused by Cytospora punicae in western Iran. New Disease Reports 35: 1-1.

Norphanphoun C, Doilom M, Daranagama DA, Phookamsak R, et al. (2017) Revisiting the genus Cytospora and allied species. Mycosphere 8: 51-97.

O'Donnell K, Ward TJ, Robert VA, Crous PW, Geiser DM, Kang S (2015) DNA sequence-based identification of Fusarium: current status and future directions. Phytoparasitica 43: 583-595.

Palavouzis S, Tzamos S, Paplomatas E, Thomidis T (2015) First report of Cytospora punicae isolated from pomegranate plants with symptom of collar rot in northern Greece. Journal of Plant Pathology 97: 209-220.

Peduto Hand F, Choudhury R, Gubler W (2014) First report of Cytospora punicae causing wood canker and branch dieback of pomegranate (Punica granatum) in the United States. Plant Disease 98: 853-853.

Rayner RW (1970) A Mycological Colour Chart. Kew: Commonwealth Mycological Institute.

Rossman AY, Adams GC, Cannon PF, Castlebury LA, Crous PW, et al. (2015) Recommendations of generic names in Diaporthales competing for protection or use. IMA Fungus 6: 145-154.

Samouel S, Kanetis L (2016) First report of Cytospora punicae causing trunk canker of pomegranate (Punica granatum) in Cyprus. Plant Disease 100: 222-222.

Schoch CL, Seifert KA, Huhndorf S, Robert V, Spouge JL, et al. (2012) Nuclear ribosomal internal transcribed spacer (ITS) region as a universal DNA barcode marker for Fungi. Proceedings of the National Academy of Sciences, USA 109: 6241-6246.

Sharma JK, Mohanan C, Florence EJM (1985) Disease survey in nurseries and plantations of forest tree species grown in Kerala. Kerala Forest Research Institute Research Report 36: 258-262.

Sinclair WA, Lyon HH, Johnson WT (1987) Diseases of Trees and Shrubs. Ithaca, NY: Cornell University Press.

Spielman LJ (1983) Taxonomy and biology of Valsa species on hardwoods of North America, with special reference to species on maples. PhD thesis, Cornell University.

Spielman LJ (1985) A monograph of Valsa on hardwoods in North America. Canadian Journal of Botany 63: 1355-1378.

Tamura K, Stecher G, Peterson D, Filipski A, Kumar S (2013) MEGA6: Molecular Evolutionary Genetics Analysis Version 6.0. Molecular Biology and Evolution 30: 2725-2729.

Tekauz A, Patrick Z (1974) The role of twig infections on the incidence of perennial canker of peach. Phytopathology 64: 683-688.

Trouillas FP, Peduto F, Lorber J, Sosnowski M, Grant J, et al. (2012) Calosphaeria canker of sweet cherry caused by Calosphaeria pulchella in California and South Australia. Plant Disease 96 648-658.

Tulasne LR, Tulasne C (1863) Selecta Fungorum Carpologia. Vol. X. [Translated by W. B. Grove.] Oxford: Clarendon Press.

Venter E, Lennox C, Meitz-Hopkins J (2017) First report of Cytospora punicae causing post-harvest fruit rot on pomegranate in South Africa. Plant Disease 101: 631-631.

Wang X, Wei J, Huang L, Kang Z (2011) Re-evaluation of pathogens causing Valsa canker on apple in China. Mycologia 103: 317324.

White TJ, Bruns T, Lee S, Taylor J (1990) Amplification and direct sequencing of fungal and ribosomal RNA genes for phylogenetics. 